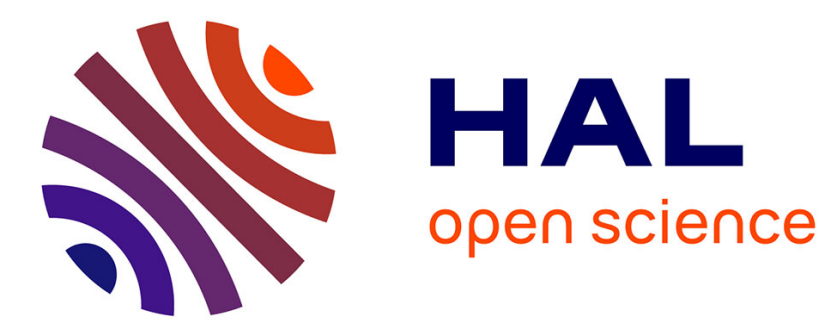

\title{
On a Kinetic Equation for Coalescing Particles
}

Miguel Escobedo, Philippe Laurençot, Stéphane Mischler

\section{To cite this version:}

Miguel Escobedo, Philippe Laurençot, Stéphane Mischler. On a Kinetic Equation for Coalescing Particles. [Research Report] RR-4748, INRIA. 2003. inria-00071839

\section{HAL Id: inria-00071839 https://hal.inria.fr/inria-00071839}

Submitted on 23 May 2006

HAL is a multi-disciplinary open access archive for the deposit and dissemination of scientific research documents, whether they are published or not. The documents may come from teaching and research institutions in France or abroad, or from public or private research centers.
L'archive ouverte pluridisciplinaire HAL, est destinée au dépôt et à la diffusion de documents scientifiques de niveau recherche, publiés ou non, émanant des établissements d'enseignement et de recherche français ou étrangers, des laboratoires publics ou privés. 
INSTITUT NATIONAL DE RECHERCHE EN INFORMATIQUE ET EN AUTOMATIQUE

\title{
On a Kinetic Equation for Coalescing Particles
}

\author{
Miguel Escobedo — Philippe Laurençot — Stéphane Mischler
}

\section{$\mathbf{N}^{\circ} 4748$}

Février 2003

THÈME 4 



\title{
RINRIA
}

\section{On a Kinetic Equation for Coalescing Particles}

\author{
Miguel Escobedd*, Philippe Laurençot $\oplus$, Stéphane Mischler 母 $^{*}$ \\ Thème 4 - Simulation et optimisation \\ de systèmes complexes \\ Projet BANG \\ Rapport de recherche $n^{\circ} 4748$ - Février 2003 - 34 pages
}

\begin{abstract}
Existence of global weak solutions to a spatially inhomogeneous kinetic model for coalescing particles is proved, each particle being identified by its mass, momentum and position. The large time convergence to zero is also shown. The cornestone of our analysis is that, for any nonnegative and convex function, the associated Orlicz norm is a Liapunov functional. Existence and asymptotic behaviour then rely on weak and strong compactness methods in $L^{1}$ in the spirit of the DiPerna-Lions theory for the Boltzmann equation.
\end{abstract}

Key-words: Existence, stability, weak and strong compactness, long time asymptotic behavior, Liapunov functionnal, kinetic coalescence, Orlicz norm, moments, entropies, spray, droplets, dynamic stellar, Manev potential, kinetic energy

\footnotetext{
* Departamento de Matemáticas, Universidad del País Vasco, Apartado 644, E-48080 Bilbao, Spain. E-mail : mtpesmam@lg.ehu.es

† Mathématiques pour l'Industrie et la Physique, CNRS UMR 5640, Université Paul Sabatier-Toulouse 3, 118 route de Narbonne, F-31062 Toulouse cedex 4, France. E-mail : laurenco@mip.ups-tlse.fr

$\ddagger$ Projet BANG, INRIA Rocquencourt B.P.105 78153 Le Chesnay CEDEX, France, Laboratoire de Mathématiques Appliquées, Université de Versailles - Saint Quentin, 45 avenue des Etats-Unis, F-78035 Versailles, France and DMA, CNRS UMR 8553, Ecole Normale Supérieure, 45 rue d'Ulm, F-75230 Paris cedex 05, France. E-mail : mischler@math.uvsq.fr
} 


\section{Une équation cinétique pour un gaz de partciules coalescentes}

Résumé : Nous démontrons l'existence globale de solutions pour une équation cinétique modélisant la coalescence de particules identifiées par leur masse, leur impulsion et leur position. Nous montrons que les solutions tendent vers 0 asymptotiquement en temps grand. Le point clef de notre analyse est la preuve que, pour toute fonction positive convexe, la norme d'Orlicz associée est une fonctionnelle de Liapunov. Les résultats d'existence et de comportement asymptotique se déduisent alors par des techniques de compacité faible et forte dans $L^{1}$ dans l'esprit de la théorie de DiPerna et Lions pour l'équation de Boltzmann.

Mots-clés : Existence, stabilité, compacité faible et forte, comportement asymptotique, fonctionnelle de Liapunov, coalescence cinétique, norme d'Orlicz, moments, entropies, gaz de gouttelettes, dynamique stellaire, potentiel de Manev, énergie cinétique 


\section{Introduction}

We consider the Cauchy problem for a kinetic equation modelling (at a mesoscopic level) the dynamic of a system of particles undergoing coalescence (or sticky) process. More precisely, describing the gas of particles by the density $f(t, x, m, p) \geq 0$ of particles with mass $m \in \mathbb{R}_{+}:=(0,+\infty)$ and momentum $p \in \mathbb{R}^{3}$ at time $t \geq 0$ and position $x \in \Omega \subset \mathbb{R}^{3}$, we study the existence and long time behaviour of solutions to the equation

$$
\begin{aligned}
\partial_{t} f+v \cdot \nabla_{x} f & =Q(f) \text { in }(0,+\infty) \times \Omega \times Y, \\
f(0) & =f^{\text {in }} \text { in } \Omega \times Y .
\end{aligned}
$$

Here and below, in order to shorten the notations, we introduce the mass-momentum variable $y:=(m, p) \in Y:=\mathbb{R}_{+} \times \mathbb{R}^{3}$ and the velocity variable $v=p / m$. The collision operator $Q(f)$ is given by $Q(f)=Q_{1}(f)-Q_{2}(f)$, where

$$
\begin{aligned}
& Q_{1}(f)(y)=\frac{1}{2} \int_{\mathbb{R}^{3}} \int_{0}^{m} a\left(y^{\prime}, y-y^{\prime}\right) f\left(y^{\prime}\right) f\left(y-y^{\prime}\right) d m^{\prime} d p^{\prime}, \\
& Q_{2}(f)(y)=\int_{\mathbb{R}^{3}} \int_{0}^{\infty} a\left(y, y^{\prime}\right) f(y) f\left(y^{\prime}\right) d m^{\prime} d p^{\prime} .
\end{aligned}
$$

The meaning of these terms is the following. Denoting by $\{y\}=(m, p)$ a particle of massmomentum $y, Q_{1}(f)(y)$ accounts for the formation of particles $\{y\}$ by coalescence of smaller ones, i.e., by the reaction

$$
\left\{y^{\prime}\right\}+\left\{y-y^{\prime}\right\} \stackrel{a\left(y^{\prime}, y-y^{\prime}\right)}{\longrightarrow}\{y\}, \quad y^{\prime}=\left(m^{\prime}, p^{\prime}\right) \in(0, m) \times \mathbb{R}^{3},
$$

and $Q_{2}(f)(y)$ describes the depletion of particles $\{y\}$ by coagulation with other particles, i.e., by the reaction

$$
\{y\}+\left\{y^{\prime}\right\} \stackrel{a\left(y, y^{\prime}\right)}{\longrightarrow}\left\{y+y^{\prime}\right\}, \quad y^{\prime} \in Y .
$$

At a microscopic level, the collision of two particles $\{y\}$ and $\left\{y^{\prime}\right\}$ leads to the formation (by coalescence) of a single particle $\left\{y^{\prime \prime}\right\}$ with rate $a\left(y, y^{\prime}\right)$, the mass and momentum being conserved during the collision. In other words,

$$
\{y\}+\left\{y^{\prime}\right\} \stackrel{a\left(y, y^{\prime}\right)}{\longrightarrow}\left\{y^{\prime \prime}\right\} \quad \text { with } \quad y^{\prime \prime}=\left(m^{\prime \prime}, p^{\prime \prime}\right)=\left(m+m^{\prime}, p+p^{\prime}\right) .
$$

In contrast to the Boltzmann equation for elastic collisions, this is an irreversible microscopic process. Observe, in particular, that it does not preserve the kinetic energy:

$$
\Phi_{k e}\left(y, y^{\prime}\right):=\frac{|p|^{2}}{2 m}+\frac{\left|p^{\prime}\right|^{2}}{2 m^{\prime}}-\frac{\left|p^{\prime \prime}\right|^{2}}{2 m^{\prime \prime}}=\frac{m m^{\prime}}{2\left(m+m^{\prime}\right)}\left|v-v^{\prime}\right|^{2} \geq 0 .
$$

Particles evolving according to these rules are met, for instance, in dense sprays involved in combustion reactions, where liquid droplets are carried by a gaseous phase and undergo 
coalescence processes (due to collisions). The dynamics of the density of liquid droplets are then described by equation (1.1) [3, 34, 35, 36, see also [10, 12, 25]. In this context, the coalescence kernel $a$ is given by

$$
a\left(y, y^{\prime}\right)=a_{H S}\left(y, y^{\prime}\right):=\left(r+r^{\prime}\right)^{2}\left|v-v^{\prime}\right|,
$$

where $r=m^{1 / 3}, r^{\prime}=\left(m^{\prime}\right)^{1 / 3}$ denote the radii of the particles and $v=p / m, v^{\prime}=p^{\prime} / m^{\prime}$, their velocities. Note that this kernel corresponds to the well-known cross section for hard spheres in the Boltzmann theory. In fact, equation (1.1) only takes into account coalescence and neglects the fragmentation of the droplets due to the action of the gas, as well as the condensation/evaporation of the droplets and the elastic (grazing) collisions of Boltzmann type. However, all these effects may be met together, for instance, in combustion theory [37. In this case, one usually considers the following equation [21]

$$
\partial_{t} g+\nabla_{x}(v g)+\nabla_{v}(\beta g)+\partial_{r}(\omega g)=Q_{\text {coll }}(g)+Q_{b r}(g),
$$

for the density $g(t, x, r, v)$ of droplets which, at time $t \geq 0$, are at the position $x \in \Omega$ with a radius $r>0$ (droplets are supposed to be spheres), and velocity $v \in \mathbb{R}^{3}$. In this equation, $\beta$ and $\omega$ denote the droplet acceleration and evaporation rate, respectively, the term $Q_{b r}(g)$ represents the effects of the break-up of the droplets due to the action of the gas, and the term $Q_{\text {coll }}(g)$ represents the effects of binary collisions between particles. It includes both elastic collisions and collisions giving rise to the coalescence of the colliding particles. Similar equations may also be found in aerosol theory [22].

Another physical situation in which coalescence occurs may be found in stellar dynamics in the modelling of clouds of particles (galaxies!) interacting by an attractive Manev pair potential $-\alpha / r-\varepsilon / r^{2}$ (see [6, 23] and the references therein). In particular, for $\alpha=0$ and $\varepsilon=1$ (Newtonian potential), the associated cross-section is

$$
a\left(y, y^{\prime}\right)=a_{N P}\left(y, y^{\prime}\right):=\frac{m+m^{\prime}}{m m^{\prime}} \frac{1}{\left|v-v^{\prime}\right|} .
$$

In both models described here, the coalescence rate $a$ corresponds to the collision frequency. But, collisions may not always result in a coagulation event. This fact can be accounted for by the introduction of a coalescence efficiency $E$ (representing the probability that the two colliding particles do really stick). Then $a\left(y, y^{\prime}\right)=E\left(y, y^{\prime}\right) a_{0}\left(y, y^{\prime}\right)$, where $a_{0}$ is the frequency of collisions and may be given, for instance, by (1.5) or (1.6), see [35].

Let us now describe the coalescence rate $a$ that we will consider in this paper. We assume that $a$ fulfils the symmetry and positivity conditions

$$
0<a\left(y, y^{\prime}\right)=a\left(y^{\prime}, y\right) \quad \text { a.e. on } Y^{2},
$$

and the structure condition

$$
a\left(y, y^{\prime}\right) \leq a\left(y, y+y^{\prime}\right)+a\left(y^{\prime}, y+y^{\prime}\right), \quad y, y^{\prime} \in Y .
$$

INRIA 
We also require the growth conditions

$$
\begin{aligned}
& a_{\delta, R}(M):=\sup _{y^{\prime} \in Y_{\delta, R}} \int_{Y_{\delta, R}} a\left(y, y^{\prime}\right) \mathbf{1}_{a\left(y, y^{\prime}\right) \geq M} d y \underset{M \rightarrow \infty}{\longrightarrow} 0, \\
& \omega_{\delta, R}\left(R^{\prime}\right):=\sup _{p^{\prime} \in \mathbb{R}^{3}, m^{\prime} \geq R^{\prime}} \int_{Y_{\delta, R}} \frac{a\left(y, y^{\prime}\right)}{\left|y^{\prime}\right|} d y \underset{R^{\prime} \rightarrow \infty}{\longrightarrow} 0,
\end{aligned}
$$

for any $R>\delta$, with $Y_{\delta, R}:=(\delta, R) \times B_{R}$. In particular, the coalescence kernel $a_{H S}$ and $a_{N P}$ fulfil the above assumptions, as it is shown in Appendix B.

As for $\Omega$, we may consider the case of the whole space $\Omega=\mathbb{R}^{3}$, the case of the 3 dimensional torus $\Omega=\mathbb{T}$ or the case of a bounded domain of $\mathbb{R}^{3}$. In the latter cases, one has to supplement (1.1) with either periodic boundary conditions or, for instance, no-incoming flux conditions

$$
\gamma f=0 \quad \text { on } \quad\{x \in \partial \Omega, n(x) \cdot v<0\},
$$

where $\gamma f$ stands for the trace of $f$ on the boundary and $n(x)$ denotes the outward normal unit vector field. In order to simplify the presentation, we only consider the case $\Omega=\mathbb{R}^{3}$ in the sequel. However, we keep the notation $\Omega$ to differentiate between the space of positions and the space of momenta (or velocities).

We finally require that the initial datum has finite total number of particles, finite total mass and finite mean momentum, that is,

$$
0 \leq f^{i n} \in L^{1}(\Omega \times Y,(1+m+|p|) d y d x) .
$$

Our main result is the following.

Theorem 1.1 Assume that the coalescence kernel a fulfils the assumptions (1.7)-(1.10) and that the initial datum satisfies (1.12). There exists at least a solution $f \geq 0$ to the coalescence equation (1.1)-(1.2) which satisfies

$$
f \in \mathcal{C}\left([0,+\infty) ; L^{1}(\Omega \times Y)\right), \quad Q(f) \in L^{1}\left(\Omega_{T} \times Y\right)
$$

for all $T>0$, where $\Omega_{T}:=(0, T) \times \Omega$. Moreover,

$$
\partial_{t} \rho+\operatorname{div}_{x} j \leq 0 \quad \text { in } \quad \mathcal{D}^{\prime}\left(\Omega_{T}\right), \quad \text { with } \quad \rho=\int_{Y} m f d y, j=\int_{Y} p f d y,
$$

and, in particular,

$$
t \longmapsto \int_{\Omega} \int_{Y} m f(t, x, y) d y d x \quad \text { is a non-increasing function of time. }
$$

Finally, the solution satisfies $f(t) \rightarrow 0$ in $L^{1}(\Omega \times Y)$ as $t \rightarrow+\infty$.

$\mathrm{RR} \mathrm{n}^{\circ} 4748$ 
Furthermore, if $f^{\text {in }} \in L^{p}(\Omega \times Y)$ for some $p \in[1, \infty]$, then

$$
t \longmapsto\|f(t)\|_{L^{p}(\Omega \times Y)} \quad \text { is a non-increasing function of time. }
$$

Finally, if supp $f^{i n} \subset \mathcal{V}_{R}=\{(x, y) \in \Omega \times Y,|v| \leq R\}$ for some $R>0$, then supp $f(t) \subset \mathcal{V}_{R}$ for any $t \geq 0$. If $f^{i n} m|v|^{2} \in L^{1}(\Omega \times Y)$ (that is, fin has finite kinetic energy), then

$$
t \longmapsto\left\|f(t) m|v|^{2}\right\|_{L^{1}(\Omega \times Y)} \quad \text { is a non-increasing function of time. }
$$

To our knowledge, the existence of solutions to the coalescence equation (1.1) has not been studied yet when the distribution function $f$ depends on the four variables $(t, x, m, p)$. In the spatially homogeneous case $f=f(t, m, p)$, existence and uniqueness of solutions are established in [35], while a numerical scheme is developed in [36]. Let us point out that, in [35, 36], the formulation of (1.1) is different and involves the variables $(r, v)$ (with $r=m^{1 / 3}$, $v=p / m)$ instead of $(m, p)$. Nevertheless, the two formulations are equivalent as it is shown in Appendix A. When the distribution function $f=f(t, m)$ does not depend on $(x, p)$, the equation (1.1) reduces to the classical Smoluchowski coagulation equation which has been extensively studied since the pioneering work of Melzak [30. We refer to the survey by Aldous [1] and the book by Dubovski [20, for a more detailed description. When the distribution function $f=f(t, x, m)$ does not depend on the momentum, a related equation, the diffusive coagulation equation, has received much attention recently. In this equation, the evolution of $f$ with respect to the position $x \in \Omega$ is modelled by a diffusion term $-d(m) \Delta_{x} f$ instead of the advection term $v \cdot \nabla_{x} f$. Since the works by Bénilan \& Wrzosek [5] and Collet \& Poupaud [11] concerning the discrete diffusive coagulation equation, existence of solutions to the continuous diffusive coagulation equation has been investigated in [2, 13, 27, 31. Let us point out here that the key estimates used in of Theorem 1.1] are in the spirit of [26, 27, 31]. We finally mention that, very recently, a kinetic equation (with velocity variable) for particles undergoing linear fragmentation has been studied in [21, 24].

Remark 1.2 On the one hand, it is likely that the existence result stated in Theorem 1.1 extends to the case where the droplets acceleration and evaporation are taken into account (that is, $\nabla_{v}(\beta f)+\partial_{m}(\omega f)$ is added to the left-hand side of (1.1)), under suitable assumptions on the acceleration and evaporation rates. Following [27], a linear fragmentation term could also be added to equation (1.1) under, for instance, an assumption of weakness of the fragmentation mechanism with respect to the coalescence mechanism. On the other hand, the present analysis does not carry over to the case where elastic collisions are also included and a collision term of Boltzmann type is added to the right-hand side of (1.1). In that case, the $L^{p}$-norms are no longer Liapunov functionals and we do not know how to remedy to this fact.

Let us now give some comments on Theorem 1.1 It turns out that Theorem 1.1 is a consequence of a stability result (see Section 3 ) which asserts that a sequence $\left(f_{n}\right)$ of solutions to (1.1) satisfying natural bounds converges weakly in $L^{1}$, up to a subsequence, to a solution to (1.1). Roughly speaking, the main mathematical difficulty is to prevent the formation of 
a Dirac mass at some point of the phase space $\Omega \times Y$. The structure assumption (1.8) allows us to prove that, for any nonnegative and convex function, the associated Orlicz norm is a Liapunov functional, which prevents concentration by the Dunford-Pettis theorem. In fact, we can prove the weak compactness in $L^{1}$ of both $\left(f_{n}\right)$ and $\left(Q\left(f_{n}\right)\right)$. Strong compactness in $L^{1}$ of $y$-averages of $f_{n}$ then follows by the velocity averaging lemma of solutions to the transport equation. The remainder of the proof is then performed in the spirit of the DiPerna-Lions theory for the Boltzmann equation [15].

Let us finally remark that, for the kernel $a_{H S}$, stationary solutions to (1.1) are

$$
S(m, p)=\mu(m) \delta_{v=u}
$$

with $\mu \in M^{1}(0,+\infty)$ is a bounded measure and $u \in \mathbb{R}^{3}$. Theorem 1.1 implies that the zero solution is the only stationary state which is reached in the long time when starting from an $L^{1}$ initial data. We thus identify more accurately the asymptotic state than in [35]. Let us also mention that, if $S_{t, x}$ is a solution to (1.1) which is a stationary solution for each $(t, x)$, that is, $S_{t, x}$ is given by (1.17) with $\mu=\mu_{t, x}$ and $u=u(t, x)$, it satisfies

$$
\partial_{t} S_{t, x}+v \cdot \nabla_{x} S_{t, x}=0 .
$$

Introducing $\varrho(t, x):=<m, \mu_{t, x}>$, we realize that $(\varrho, u)$ satisfies the pressureless gases system

$$
\partial_{t} \varrho+\partial_{x}(\varrho u)=0, \quad \partial_{t}(\varrho u)+\partial_{x}\left(\varrho u^{2}\right)=0,
$$

see [8, 9, 38] and the references therein.

We now outline the contents of this paper. In the next section, we collect some qualitative and formal information on the solutions. They lead to the natural bounds that one can expect on the solutions. In Section 3 we specify the notion of solution we deal with, and state the key stability theorem (Theorem 3.6) which is proved in Section 4. Section 5 is devoted to the proof of the convergence of the solutions to zero for large times. We then briefly explain in Section 6 how the stability result adapts to prove Theorem 1.1 We finally establish the equivalence between the two formulations radius-velocity $(r, v)$ and mass-momentum $(m, p)$ of (1.1) in Appendix A and then check that the coalescence kernels $a_{H S}$ and $a_{N P}$ given by (1.5) and (1.6) do satisfy (1.7)-(1.10) in Appendix B.

Acknowledgements. We gratefully acknowledge the partial support of the European Research Training Network HYKE HPRN-CT-2002-00282 during this work. The first and third authors were partially supported by CNRS and UPV/EHU through a PICS between the Universidad del País Vasco and the Ecole Normale Supérieure. We also thank Pierre Degond for fruitful discussions which motivate us to extend Theorem 1.1 to initial data with possibly infinite kinetic energy. 


\section{Conservation laws and Liapunov functionals}

In this section we derive some (formal) conserved quantities and non-increasing ones as well.

Let us start with the following fundamental (and formal) identity: for any $\phi: Y \rightarrow \mathbb{R}_{+}$ there holds

$$
\int_{Y} Q(f) \phi d y=\frac{1}{2} \int_{Y} \int_{Y} a\left(y, y^{\prime}\right) f f^{\prime}\left(\phi^{\prime \prime}-\phi-\phi^{\prime}\right) d y^{\prime} d y .
$$

This identity is obtained after changing variables and applying (without justification) the Fubini theorem to $Q_{1}(f)$. Here and below, we put $g=g(y), g^{\prime}=g\left(y^{\prime}\right)$ and $g^{\prime \prime}=g\left(y+y^{\prime}\right)$ to shorten notations.

Suitable choices of functions $\phi$ in (2.19) lead to several qualitative information on the solution $f$ to the coalescence equation (1.1), and on the reaction term $Q(f)$ as well. We list some of them now.

- Mass conservation. With the choice $\phi(y)=m$, the term $\phi^{\prime \prime}-\phi-\phi^{\prime}$ vanishes and we deduce the total mass conservation:

$$
\int_{\Omega} \int_{Y} m f(t, x, y) d y d x=\int_{\Omega} \int_{Y} m f^{i n}(x, y) d y d x, \quad t \geq 0 .
$$

- Momentum conservation. Similarly, with the choice $\phi(y)=p$, the term $\phi^{\prime \prime}-\phi-\phi^{\prime}$ also vanishes and we deduce the mean momentum conservation:

$$
\int_{\Omega} \int_{Y} p f(t, x, y) d y d x=\int_{\Omega} \int_{Y} p f^{i n}(x, y) d y d x, \quad t \geq 0 .
$$

It is next clear from (2.19) that, if $\phi: Y \rightarrow \mathbb{R}$ is subadditive, that is,

$$
\phi\left(y+y^{\prime}\right) \leq \phi(y)+\phi\left(y^{\prime}\right) \text { for all }\left(y, y^{\prime}\right) \in Y^{2},
$$

the following map

$$
t \mapsto \int_{\Omega} \int_{Y} f(t, x, y) \phi(y) d y d x
$$

is a non-increasing function of time. We now identify several classes of functions satisfying $(2.22)$ : we first consider functions depending solely on $m$, then functions depending solely on $v$, and finally functions being the product of a function of $m$ and a function of $v$.

- Typical examples of subadditive functions is $\phi(y)=m^{\alpha}$ for $\alpha \in(-\infty, 1]$. In particular, the choice $\phi(y)=1$ shows that the so-called total number of particles decreases with time (as expected)

$$
\int_{\Omega} \int_{Y} f(t, x, y) d y d x+\frac{1}{2} \int_{0}^{t} \int_{\Omega} \int_{Y} \int_{Y} a f f^{\prime} d y^{\prime} d y d x d t=\int_{\Omega} \int_{Y} f^{i n}(x, y) d y d x .
$$


Another consequence is that, if $\operatorname{supp} f^{i n}(x, y) \subset \mathcal{M}_{\delta}:=\{(x, y) \in \Omega \times Y, m>\delta\}$, then $\operatorname{supp} f(t) \subset \mathcal{M}_{\delta}$ for any $t \geq 0$. Indeed, it is sufficient to notice that $\phi(y)=\mathbf{1}_{[0, \delta]}(m)$ satisfies (2.22). That means that no particle of size smaller than $\delta$ can be created if there are none initially. A weaker version of this fact is the following: for any non-increasing function $\phi: \mathbb{R}_{+} \rightarrow \mathbb{R}_{+}$(such as $\left.\phi(m)=m^{\alpha}, \alpha<0\right)$, we have

$$
Y_{\phi}(f(t, .))+\frac{1}{2} \int_{0}^{t} \int_{\Omega} \mathcal{Y}_{\phi}(f(\tau)) d x d \tau \leq Y_{\phi}\left(f^{i n}\right)
$$

with

$$
\begin{aligned}
Y_{\phi}(f) & :=\int_{\Omega} \int_{Y} f(x, y) \phi(m) d y d x \\
\mathcal{Y}_{\phi}(f) & :=\int_{Y} \int_{Y} a \phi(m) f f^{\prime} d y d x .
\end{aligned}
$$

- For any non-decreasing and nonnegative function $\phi: \mathbb{R}_{+} \rightarrow \mathbb{R}_{+}$, we notice that

$$
\left|p+p^{\prime}\right| \leq|p|+\left|p^{\prime}\right| \leq\left(m+m^{\prime}\right) \max \left(|v|,\left|v^{\prime}\right|\right),
$$

and thus $\left|v^{\prime \prime}\right| \leq \max \left(|v|,\left|v^{\prime}\right|\right)$. The monotonicity of $\phi$ then implies that $\phi$ fulfils (2.22). Consequently,

$$
t \longmapsto \int_{\Omega} \int_{Y} f(t, x, y) \phi(|v|) d y d x
$$

is a non-increasing function of time. As a first consequence of this fact, we realize that, if $\operatorname{supp} f^{i n} \subset \mathcal{V}_{R}:=\{(x, y) \in \Omega \times Y,|v| \leq R\}$, then supp $f(t) \subset \mathcal{V}_{R}$ for any $t \geq 0$ (apply the previous result with the choice $\left.\phi=\mathbf{1}_{[R,+\infty)}\right)$.

- Consider now a nonnegative and non-increasing function $\phi_{1}: \mathbb{R}_{+} \rightarrow \mathbb{R}$ and a nonnegative and convex function $\phi_{2}: \mathbb{R}^{3} \rightarrow \mathbb{R}$. Then

$$
t \longmapsto \int_{\Omega} \int_{Y} f(t, x, y) m \phi_{1}(m) \phi_{2}(v) d y d x
$$

is a non-increasing function of time. Indeed, putting $\phi(y)=m \phi_{1}(m) \phi_{2}(p / m), y \in Y$, the convexity of $\phi_{2}$ and the monotonicity of $\phi_{1}$ ensure that

$$
\begin{aligned}
\phi\left(y+y^{\prime}\right) & =\left(m+m^{\prime}\right) \phi_{1}\left(m+m^{\prime}\right) \phi_{2}\left(\frac{m}{m+m^{\prime}} v+\frac{m^{\prime}}{m+m^{\prime}} v^{\prime}\right) \\
& \leq \phi_{1}\left(m+m^{\prime}\right)\left(m \phi_{2}(v)+m^{\prime} \phi_{2}\left(v^{\prime}\right)\right) \\
& \leq m \phi_{1}(m) \phi_{2}(v)+m^{\prime} \phi_{1}\left(m^{\prime}\right) \phi_{2}\left(v^{\prime}\right),
\end{aligned}
$$

and the function $\phi$ satisfies (2.22). 
A first interesting consequence of this result is the decay of the kinetic energy (with the choice $\phi(y)=|p|^{2} / m$, that is, $\phi_{1}(m)=1$ and $\left.\phi_{2}(v)=|v|^{2}\right)$ :

$$
\int_{\Omega} \int_{Y} f(t, x, y) \frac{|p|^{2}}{m} d y d x+\frac{1}{2} \int_{0}^{t} \int_{\Omega} \mathcal{E}_{k e}(f(t, x, .)) d x d t=\int_{\Omega} \int_{Y} f^{i n}(x, y) \frac{|p|^{2}}{m} d y d x,
$$

where

$$
\mathcal{E}_{k e}(f):=\int_{Y} \int_{Y} a \frac{m m^{\prime}}{m+m^{\prime}}\left|v-v^{\prime}\right|^{2} f f^{\prime} d y^{\prime} d y .
$$

A more general property is actually valid. Consider a nonnegative and non-decreasing convex function $\lambda \in \mathcal{C}^{1}([0,+\infty))$ such that $\lambda(0)=0$. With the choice $\phi(y)=m \lambda(|p| / m)$ (that is, $\phi_{1}(m)=1$ and $\phi_{2}(v)=\lambda(|v|)$ ), the function $\phi$ is subadditive and we have

$$
\int_{\Omega} \int_{Y} f(t, x, y) m \lambda(|v|) d y d x+\frac{1}{2} \int_{0}^{t} \int_{\Omega} \mathcal{E}_{\lambda}(f(t, x)) d x d t \leq \int_{\Omega} \int_{Y} f^{i n}(x, y) m \lambda(|v|) d y d x,
$$

where

$$
\mathcal{E}_{\lambda}(f):=\int_{Y} \int_{Y} a m \Lambda\left(\frac{|p|}{m}, \frac{|p|+\left|p^{\prime}\right|}{m+m^{\prime}}\right) f f^{\prime} d y^{\prime} d y, \quad \Lambda(A, B):=\int_{A}^{B}\left(\lambda^{\prime}(B)-\lambda^{\prime}(z)\right) d z .
$$

Indeed, putting $w=\left(|p|+\left|p^{\prime}\right|\right) /\left(m+m^{\prime}\right)$, the monotonicity of $\lambda$ entails that

$$
\phi(y)+\phi\left(y^{\prime}\right)-\phi\left(y+y^{\prime}\right) \geq m^{\prime}\left(\lambda\left(\left|v^{\prime}\right|\right)-\lambda(w)\right)+m(\lambda(|v|)-\lambda(w)) .
$$

The convexity of $\lambda$ and the identity $m^{\prime}\left(\left|v^{\prime}\right|-w\right)=m(w-|v|)$ then imply

$$
\begin{aligned}
\phi(y)+\phi\left(y^{\prime}\right)-\phi\left(y+y^{\prime}\right) & \geq m^{\prime}\left(\left|v^{\prime}\right|-w\right) \lambda^{\prime}(w)-m \int_{|v|}^{w} \lambda^{\prime}(z) d z \\
& \geq m \int_{|v|}^{w}\left(\lambda^{\prime}(w)-\lambda^{\prime}(z)\right) d z=\Lambda(|v|, w) .
\end{aligned}
$$

Another consequence is the following. For any convex function $\omega: \mathbb{R}^{3} \rightarrow \mathbb{R}_{+}$, the function

$$
t \longmapsto \int_{\Omega} \int_{Y} f(t, x, y) \omega(x-v t) d y d x
$$

is a non-increasing function of time. Indeed, for each $(t, x) \in \mathbb{R}_{+} \times \mathbb{R}^{3}, \phi: y \mapsto \omega(x-v t)$ is a convex function of $v$ and is thus subadditive. Therefore, after multiplying (1.1) by $\omega(x-v t)$ and integration, the contribution of the coalescence term is nonnegative, while the terms resulting from the free transport cancel. In particular, taking $\omega(z)=|z|^{\theta}, \theta \geq 1, z \in \mathbb{R}^{3}$, we obtain

$$
\int_{\Omega} \int_{Y} f(t, x, y)|x-v t|^{\theta} d y d x \leq \int_{\Omega} \int_{Y} f^{i n}(x, y)|x|^{\theta} d y d x, \quad t \geq 0 .
$$


- Finally, let $\Phi:[0,+\infty) \rightarrow[0,+\infty)$ be a nonnegative and convex function satisfying $\Phi(0)=0$. Then

$$
t \longmapsto \int_{\Omega} \int_{Y} \Phi(f(t, x, y)) d y d x
$$

is a non-increasing function of time. More precisely, we have the following result

Lemma 2.3 Any solution $f$ to (1.1)-(1.2) satisfies

$$
\int_{\Omega} \int_{Y} \Phi(f(t, x, y)) d y d x+\int_{0}^{t} \int_{\Omega} D_{\Phi}(f(\tau, x)) d x d \tau \leq \int_{\Omega} \int_{Y} \Phi\left(f^{i n}(x, y)\right) d y d x,
$$

where $D_{\Phi}(f):=D_{\Phi}^{1}(f)+D_{\Phi}^{2}(f)$,

$$
\begin{aligned}
& D_{\Phi}^{1}(f):=\frac{1}{2} \int_{Y^{2}} a\left(y, y^{\prime}\right)\left(f \vee f^{\prime}\right) \Phi\left(f \wedge f^{\prime}\right) d y d y^{\prime} \geq 0, \\
& D_{\Phi}^{2}(f):=\int_{Y^{2}} a\left(y, y^{\prime}\right) \Psi(f) f^{\prime} \mathbf{1}_{(m,+\infty) \times \mathbb{R}^{3}}\left(y^{\prime}\right) d y^{\prime} d y \geq 0,
\end{aligned}
$$

and $\Psi(u):=u \Phi^{\prime}(u)-\Phi(u) \geq 0$ for $u \geq 0$. Here and below, we use the notations $f \vee f^{\prime}=$ $\max \left\{f, f^{\prime}\right\}$ and $f \wedge f^{\prime}=\min \left\{f, f^{\prime}\right\}$.

When $\Phi(u)=u^{p}, p>1$, this result has been proved in [31] for the Smoluchowski equation.

Proof. We first recall that the convex conjugate $\Phi^{*}$ of $\Phi$ is a nonnegative convex function given by

$$
\Phi^{*}(u):=\sup _{w \geq 0}\{u w-\Phi(w)\}
$$

for $u \geq 0$. In addition, we have the Young inequality

$$
u w \leq \Phi(u)+\Phi^{*}(w), \quad u, w \geq 0,
$$

and the inequality

$$
\Phi^{*}\left(\Phi^{\prime}(u)\right) \leq \Psi(u), \quad u \geq 0 .
$$

By (2.32) and (2.33) we have

$$
\begin{aligned}
\int_{Y^{2}} a f f^{\prime} \Phi^{\prime}\left(f^{\prime \prime}\right) d y^{\prime} d y & =\int_{Y^{2}} a\left(f \wedge f^{\prime}\right)\left(f \vee f^{\prime}\right) \Phi^{\prime}\left(f^{\prime \prime}\right) d y^{\prime} d y \\
& \leq \int_{Y^{2}} a\left(f \wedge f^{\prime}\right)\left\{\Phi\left(f \vee f^{\prime}\right)+\Phi^{*}\left(\Phi^{\prime}\left(f^{\prime \prime}\right)\right)\right\} d y^{\prime} d y \\
& \leq \int_{Y^{2}} a\left(f \wedge f^{\prime}\right) \Phi\left(f \vee f^{\prime}\right) d y^{\prime} d y+\int_{Y^{2}} a\left(f \wedge f^{\prime}\right) \Psi\left(f^{\prime \prime}\right) d y^{\prime} d y .
\end{aligned}
$$


We now use (1.8) to bound the second term of the right-hand side of the above inequality and deduce that

$$
\begin{aligned}
\int_{Y^{2}} a f f^{\prime} \Phi^{\prime}\left(f^{\prime \prime}\right) d y^{\prime} d y \leq & \int_{Y^{2}} a\left(f \wedge f^{\prime}\right) \Phi\left(f \vee f^{\prime}\right) d y^{\prime} d y \\
& +\int_{Y^{2}}\left(a\left(y, y^{\prime \prime}\right)+a\left(y^{\prime}, y^{\prime \prime}\right)\right)\left(f \wedge f^{\prime}\right) \Psi\left(f^{\prime \prime}\right) d y^{\prime} d y \\
\leq & \int_{Y^{2}} a\left(f \wedge f^{\prime}\right) \Phi\left(f \vee f^{\prime}\right) d y^{\prime} d y+2 \int_{Y^{2}} a\left(y^{\prime}, y^{\prime \prime}\right) f^{\prime} \Psi\left(f^{\prime \prime}\right) d y^{\prime} d y \\
\leq & \int_{Y^{2}} a\left(f \wedge f^{\prime}\right) \Phi\left(f \vee f^{\prime}\right) d y^{\prime} d y \\
& +2 \int_{Y^{2}} a f^{\prime} \Psi(f) \mathbf{1}_{(0, m) \times \mathbb{R}^{3}}\left(y^{\prime}\right) d y^{\prime} d y .
\end{aligned}
$$

Consequently,

$$
\begin{aligned}
\int_{Y} Q(f) \Phi^{\prime}(f) d y= & \frac{1}{2} \int_{Y^{2}} a f f^{\prime}\left(\Phi^{\prime}\left(f^{\prime \prime}\right)-\Phi^{\prime}(f)-\Phi^{\prime}\left(f^{\prime}\right)\right) d y^{\prime} d y \\
\leq & \frac{1}{2} \int_{Y^{2}} a\left(f \wedge f^{\prime}\right) \Phi\left(f \vee f^{\prime}\right) d y^{\prime} d y+\int_{Y^{2}} a f^{\prime} \Psi(f) \mathbf{1}_{(0, m) \times \mathbb{R}^{3}}\left(y^{\prime}\right) d y^{\prime} d y \\
& -\int_{Y^{2}} a \Psi(f) f^{\prime} d y^{\prime} d y-\frac{1}{2} \int_{Y^{2}} a\left(\Phi(f) f^{\prime}+\Phi\left(f^{\prime}\right) f\right) d y^{\prime} d y,
\end{aligned}
$$

whence (2.30).

We summarize, in the next result, the a priori estimates on the solutions to (1.1) obtained in this section.

Theorem 2.4 Assume that $f^{\text {in }}$ satisfies

$K\left(f^{i n}\right):=\int_{\Omega} \int_{Y}\left(\Phi\left(f^{i n}(x, y)\right)+f^{i n}(x, y)\left(r(m)+1+m+|p|+m \lambda\left(\frac{|p|}{m}\right)\right)\right) d x d y<\infty$,

for some nonnegative and non-decreasing convex functions $\Phi \in \mathcal{C}^{1}([0,+\infty))$ and $\lambda \in \mathcal{C}^{1}([0,+\infty))$ such that $\Phi(0)=0$ and $\lambda(0)=0$, and some nonnegative and non-increasing function $r \in \mathcal{C}((0,+\infty))$. Then a solution $f$ to (1.1)-(1.2) formally satisfies

$$
\begin{gathered}
\sup _{t \geq 0} \int_{\Omega} \int_{Y}\left(\Phi(f(t, x, y))+f(t, x, y)\left(r(m)+1+m+|p|+m \lambda\left(\frac{|p|}{m}\right)\right)\right) d x d y \leq K\left(f^{i n}\right), \\
\int_{0}^{\infty} \int_{\Omega}\left(D_{\Phi}(f(\tau, x))+\mathcal{Y}_{1}(f(\tau, x))+\mathcal{Y}_{r}(f(\tau, x))+\mathcal{E}_{\lambda}(f(\tau, x))\right) d x d \tau \leq 2 K\left(f^{i n}\right), \quad(2.36)
\end{gathered}
$$

where $\mathcal{Y}_{1}, \mathcal{Y}_{r}, \mathcal{E}_{\lambda}$ and $D_{\Phi}$ are defined in (2.24) (with $\phi=1$ and $\phi=r$, respectively), (2.28) and (2.30), respectively. 


\section{Stability result}

Definition 3.5 Let $f^{\text {in }}$ be a nonnegative function satisfying (1.12). A weak solution to (1.1)-(1.2) is a nonnegative function

$$
f \in \mathcal{C}\left([0,+\infty) ; L^{1}(\Omega \times Y)\right), \quad f(0)=f^{i n},
$$

satisfying (2.35), (2.36) for some nonnegative and non-decreasing convex functions $\Phi \in$ $\mathcal{C}^{1}([0,+\infty))$ and $\lambda \in \mathcal{C}^{1}([0,+\infty))$, and some nonnegative, convex and non-increasing function $r \in \mathcal{C}^{1}((0,+\infty))$ such that $\Phi(0)=0, \lambda(0)=0$,

$$
\lim _{u \rightarrow+\infty} \lambda^{\prime}(u)=\lim _{u \rightarrow+\infty} \frac{\Phi(u)}{u}=+\infty \quad \text { and } \quad \lim _{m \rightarrow 0} r(m)=+\infty,
$$

together with the equation (1.1) in the sense of distributions. It must also satisfy the qualitative properties (1.13)-(1.16). Notice that the bounds (2.35), (2.36) imply that equation (1.1) makes sense since, for any $T \in \mathbb{R}_{+}$,

$$
Q_{i}(f) \in L^{1}\left(\Omega_{T} \times Y\right), \quad i=1,2 .
$$

We now state a weak stability principle for weak solutions to (1.1)-(1.2).

Theorem 3.6 For $n \geq 1$, let $f_{n}$ be a weak solution to (1.1)-(1.2) with initial datum $f_{n}(0)$. Assume further that (2.35) and (2.36) hold uniformly with respect to $n \geq 1$ with $K\left(f_{n}(0)\right) \leq$ $K_{0}$ for some $K_{0}>0$ and functions $(\Phi, \lambda, r)$ satisfying (3.37). Then there exist a subsequence $\left(f_{n_{k}}\right)$ of $\left(f_{n}\right)$ and a function $f$ such that $f$ is a weak solution to (1.1)-(1.2),

$$
\left\{\begin{array}{l}
f_{n_{k}} \longrightarrow f \quad \text { in } \quad \mathcal{C}\left([0, T) ; w-L^{1}\left(B_{R} \times Y\right)\right), \\
Q_{i}\left(f_{n_{k}}\right) \rightarrow Q_{i}(f) \quad \text { in } \quad L^{1}\left((0, T) \times B_{R} \times Y_{R}\right)
\end{array}\right.
$$

for any $T, R \in \mathbb{R}_{+}$and $i \in\{1,2\}$, with $Y_{R}:=(0, R) \times B_{R}$, and

$$
\int_{Y} \psi(y) f_{n_{k}} d y \longrightarrow \int_{Y} \psi(y) f d y \quad \text { in } \quad L^{1}\left((0, T) \times B_{R}\right)
$$

for $\psi \in \mathcal{D}(Y)$. Here $\mathcal{D}(Y)$ denotes the space of $\mathcal{C}^{\infty}$-smooth and compactly supported functions in $Y$ and $\mathcal{C}\left([0, T) ; w-L^{1}(\Omega \times Y)\right)$ the space of weakly continuous functions from $[0, T)$ in $L^{1}(\Omega \times Y)$.

\section{Proof of Theorem 3.6}

In this section, we consider a sequence $\left(f_{n}\right)$ of solutions to (1.1) satisfying the requirements of Theorem 3.6. We first prove that (2.35) and (2.36) guarantee the weak compactness in $L^{1}$ of $\left(f_{n}\right)$ and $\left(Q_{i}\left(f_{n}\right)\right), i=1,2$. The next step is to use the properties of the linear transport equation to obtain the strong $L^{1}$-compactness of $y$-averages in a way similar to the Boltzmann equation [15. Thanks to the strong compactness thus obtained, we may identify the limit of the nonlinear coagulation term $\left(Q\left(f_{n}\right)\right)$. 


\subsection{Weak compactness of the coalescence term}

We first prove that (2.35) and (2.36) imply the weak $L^{1}$-compactness of $\left(f_{n}\right)$ and $\left(Q_{1}\left(f_{n}\right)\right)$.

Lemma 4.7 For each $T>0$ and $R>0$, the sequence $\left(f_{n}\right)$ is weakly compact in $L^{1}((0, T) \times$ $\left.B_{R} \times Y\right)$ and the sequence $\left(Q_{1}\left(f_{n}\right)\right)$ is weakly compact in $L^{1}\left((0, T) \times B_{R} \times Y_{R}\right)$, where $Y_{R}$ is defined in (3.39).

Proof. We fix $T>0$ and $R>0$. Observe first that the first assertion of Lemma 4.7 is a straightforward consequence of [2.35), (3.37) and the Dunford-Pettis theorem.

We next study the weak compactness properties of $\left(Q_{1}\left(f_{n}\right)\right)$. Let $E$ be a measurable subset of $(0, T) \times B_{R} \times Y_{R}$ and let $M, N, \delta$ and $R_{1}$ be positive real numbers such that $R_{1} \geq R+2 R^{2} / \delta$. Putting $\varphi=\mathbf{1}_{E}$ and

$$
A=A_{n}\left(t, x, y, y^{\prime}\right):=a\left(y, y^{\prime}\right) f_{n}(t, x, y) f_{n}\left(t, x, y^{\prime}\right),
$$

and performing the change of variables $\left(y, y^{\prime}\right) \rightarrow\left(y^{\prime}, y-y^{\prime}\right)$, we have

$$
\int_{Y_{R}} Q_{1}\left(f_{n}\right) \varphi d y=\mathcal{I}_{1}+\mathcal{J}_{1}
$$

with

$$
\begin{aligned}
2 \mathcal{I}_{1}= & \int_{0}^{\delta} \int_{\mathbb{R}^{3}} \int_{0}^{R} \int_{B\left(-p^{\prime}, R\right)} A \varphi^{\prime \prime} d y d y^{\prime}+\int_{\delta}^{R} \int_{\mathbb{R}^{3}} \int_{0}^{\delta} \int_{B\left(-p^{\prime}, R\right)} A \varphi^{\prime \prime} d y d y^{\prime} \\
& +\int_{\delta}^{R} \int_{\left\{\left|p^{\prime}\right|>R_{1}\right\}} \int_{\delta}^{R} \int_{B\left(-p^{\prime}, R\right)} A \varphi^{\prime \prime} d y d y^{\prime} \\
& +\int_{\delta}^{R} \int_{\left\{\left|p^{\prime}\right| \leq R_{1}\right\}} \int_{\delta}^{R} \int_{B\left(-p^{\prime}, R\right)} A \varphi^{\prime \prime} \mathbf{1}_{a \geq N} d y d y^{\prime}
\end{aligned}
$$

and

$$
\mathcal{J}_{1}=\frac{1}{2} \int_{\delta}^{R} \int_{\left\{\left|p^{\prime}\right| \leq R_{1}\right\}} \int_{\delta}^{R} \int_{B\left(-p^{\prime}, R\right)} A \varphi^{\prime \prime} \mathbf{1}_{a \leq N} d y d y^{\prime} .
$$

On the one hand, observing that

$$
\frac{|p|}{m} \leq \frac{R}{\delta} \leq \frac{R_{1}}{2 R} \leq \frac{\left|p^{\prime}\right|+|p|}{m+m^{\prime}},
$$

so that

$$
m \Lambda\left(\frac{|p|}{m}, \frac{\left|p^{\prime}\right|+|p|}{m+m^{\prime}}\right) \geq \Lambda_{\delta, R}\left(R_{1}\right):=\delta \Lambda\left(\frac{R}{\delta}, \frac{R_{1}}{2 R}\right)
$$

INRIA 
for $y \in(\delta, R) \times B\left(-p^{\prime}, R\right)$ and $y^{\prime} \in(\delta, R) \times B_{R_{1}}^{c}$, and

$$
\begin{aligned}
A & =a\left(f_{n} \vee f_{n}^{\prime}\right)\left(f_{n} \wedge f_{n}^{\prime}\right) \\
& \leq a\left(f_{n} \vee f_{n}^{\prime}\right) M \mathbf{1}_{f_{n} \wedge f_{n}^{\prime} \leq M}+\frac{M}{\Phi(M)} a\left(f_{n} \vee f_{n}^{\prime}\right) \Phi\left(f_{n} \wedge f_{n}^{\prime}\right) \mathbf{1}_{f_{n} \wedge f_{n}^{\prime} \geq M} \\
& \leq a\left(f_{n}+f_{n}^{\prime}\right) M+\frac{M}{\Phi(M)} a\left(f_{n} \vee f_{n}^{\prime}\right) \Phi\left(f_{n} \wedge f_{n}^{\prime}\right),
\end{aligned}
$$

we get

$$
\begin{aligned}
2 \mathcal{I}_{1} \leq & \frac{1}{r(\delta)} \int_{0}^{\delta} \iint_{0}^{R} \int_{B\left(-p^{\prime}, R\right)} r(m) A d y d y^{\prime}+\frac{1}{r(\delta)} \int_{\delta}^{R} \iint_{0}^{\delta} \int_{B\left(-p^{\prime}, R\right)} r\left(m^{\prime}\right) A d y d y^{\prime} \\
& +\frac{1}{\Lambda_{\delta, R}\left(R_{1}\right)} \int_{\delta}^{R} \int_{\left\{\left|p^{\prime}\right|>R_{1}\right\}} \int_{\delta}^{R} \int_{B\left(-p^{\prime}, R\right)} m \Lambda\left(\frac{|p|}{m}, \frac{|p|+\left|p^{\prime}\right|}{m+m^{\prime}}\right) A d y d y^{\prime} \\
& +M \iint_{Y_{\delta, R+R_{1}}^{2}} a \mathbf{1}_{a \geq N}\left(f_{n}+f_{n}^{\prime}\right) d y d y^{\prime} \\
& +\frac{M}{\Phi(M)} \iint_{Y_{\delta, R+R_{1}}} a\left(f_{n} \vee f_{n}^{\prime}\right) \Phi\left(f_{n} \wedge f_{n}^{\prime}\right) d y d y^{\prime} \\
\leq & \frac{2}{r(\delta)} \mathcal{Y}_{r}\left(f_{n}(t, x)\right)+\frac{1}{\Lambda_{\delta, R}\left(R_{1}\right)} \mathcal{E}_{\lambda}\left(f_{n}(t, x)\right) \\
& +2 M a_{\delta, R+R_{1}}(N) \int_{Y} f_{n}(t, x) d y+2 \frac{M}{\Phi(M)} D_{\Phi}^{1}\left(f_{n}(t, x)\right) .
\end{aligned}
$$

On the other hand, by (1.9) and (4.42), we have

$$
\begin{aligned}
\mathcal{J}_{1} & \leq \int_{Y_{\delta, R+R_{1}}} \int_{Y_{\delta, R+R_{1}}} A \mathbf{1}_{a \leq N} \varphi^{\prime \prime} d y d y^{\prime} \\
& \leq M N \int_{Y_{\delta, R+R_{1}}} \int_{Y_{\delta, R+R_{1}}}\left(f_{n}+f_{n}^{\prime}\right) \varphi^{\prime \prime} d y d y^{\prime}+\frac{2 M}{\Phi(M)} D_{\Phi}^{1}\left(f_{n}(t, x)\right) \\
& \leq 2 M N \int_{Y^{2}} f_{n} \varphi^{\prime} d y^{\prime} d y+\frac{2 M}{\Phi(M)} D_{\Phi}^{1}\left(f_{n}(t, x)\right) .
\end{aligned}
$$

We now gather the previous estimates and integrate over $(0, T) \times B_{R}$. Owing to 2.35 and (2.36), we end up with

$$
\begin{aligned}
& 2 \int_{0}^{T} \int_{B_{R}} \int_{Y_{R}} Q_{1}\left(f_{n}\right) \varphi d y d x d t \leq \frac{2 K_{0}}{r(\delta)}+\frac{K_{0}}{\Lambda_{\delta, R}\left(R_{1}\right)}+\frac{4 M}{\Phi(M)} K_{0} \\
& \quad+2 M a_{\delta, R+R_{1}}(N) K_{0}+M N \int_{0}^{T} \int_{B_{R}} \int_{Y^{2}} f_{n} \varphi^{\prime} d y^{\prime} d y d x d t
\end{aligned}
$$

RR $\mathrm{n}^{\circ} 4748$ 
Using the already established weak compactness of $\left(f_{n}\right)$ in $L^{1}\left((0, T) \times B_{R} \times Y\right)$ and (1.9), we may pass to the limit first as $|E| \rightarrow 0$, then as $N \rightarrow+\infty, R_{1} \rightarrow+\infty$, and finally as $M \rightarrow+\infty$ and $\delta \rightarrow 0$ to conclude that

$$
\lim _{|E| \rightarrow 0} \sup _{n \geq 1} \int_{0}^{T} \int_{B_{R}} \int_{Y_{R}} Q_{1}\left(f_{n}\right) \mathbf{1}_{E} d y d x d t=0,
$$

after noticing that 3.37 implies that $\Lambda_{\delta, R}\left(R_{1}\right) \rightarrow+\infty$ as $R_{1} \rightarrow+\infty$. Therefore, $\left(Q_{1}\left(f_{n}\right)\right)$ is weakly compact in $L^{1}\left((0, T) \times B_{R} \times Y_{R}\right)$ by the Dunford-Pettis theorem.

Owing to the structure of the coalescence equation (1.1), it turns out that the weak $L^{1}$-compactness of $\left(Q_{1}\left(f_{n}\right)\right)$ provides some additional information on $\left(Q_{2}\left(f_{n}\right)\right)$. This fact was first observed in [26] for the discrete diffusive coagulation equation and a result in the same spirit is also available for (1.1).

Lemma 4.8 For any $R, T>0$, there exists a nonnegative and non-decreasing function $\theta=\theta_{R, T} \in \mathcal{C}^{1}([0,+\infty))$ such that

$$
\theta^{\prime}(u) \rightarrow+\infty \quad \text { when } \quad u \rightarrow+\infty
$$

and

$$
\int_{0}^{T} \int_{B_{R}} \int_{Y_{\delta, R}} \theta^{\prime}\left(f_{n}\right) Q_{2}\left(f_{n}\right) d y d x d t \leq C(\delta, R, T)
$$

for any $\delta \in(0,1)$.

Proof. From Lemma 4.7, we know that $\left(f_{n}(0)\right),\left(f_{n}\right)$ and $\left(Q_{1}\left(f_{n}\right)\right)$ belong to weakly compact subsets of $L^{1}(\Omega \times Y)$ and $L^{1}\left((0, T) \times B_{2 R} \times Y_{2 R}\right)$, respectively. The Dunford-Pettis theorem and a refined version of the de la Vallée-Poussin theorem [28, Proposition I.1.1] imply that there is a nonnegative, convex and non-decreasing function $\theta=\theta_{R, T} \in \mathcal{C}^{1}([0,+\infty))$ such that

$$
\theta^{\prime}(u) \rightarrow+\infty \quad \text { as } \quad u \rightarrow+\infty \quad \text { and } \quad u \theta^{\prime}(u) \leq C_{\theta} \theta(u), \quad u \geq 0,
$$

for some constant $C_{\theta}>1$ and

$$
K_{1}:=\sup _{n \geq 1}\left\{\int_{B_{2 R}} \int_{Y_{2 R}} \theta\left(f_{n}(0)\right) d y d x+\int_{0}^{T} \int_{B_{2 R}} \int_{Y_{2 R}}\left(\theta\left(f_{n}\right)+\theta\left(Q_{1}\left(f_{n}\right)\right)\right) d y d x d t\right\}<+\infty .
$$

We fix $\chi \in \mathcal{D}\left(\mathbb{R}^{3} \times Y\right), 0 \leq \chi \leq 1$, such that $\chi \equiv 1$ on $B_{R} \times Y_{\delta, R}$ and $\operatorname{supp} \chi \subset$ $B_{2 R} \times Y_{\delta / 2,2 R}$. We infer from (1.1) that

$$
\begin{aligned}
& \int_{\Omega} \int_{Y} \theta\left(f_{n}(T)\right) \chi d y d x+\int_{\Omega_{T}} \int_{Y} \theta^{\prime}\left(f_{n}\right) Q_{2}\left(f_{n}\right) \chi d y d x d t \\
& \quad=\int_{\Omega_{T}} \int_{Y} \theta^{\prime}\left(f_{n}\right) Q_{1}\left(f_{n}\right) \chi d y d x d t+\int_{\Omega_{T}} \int_{Y} \theta\left(f_{n}\right) v \cdot \nabla_{x} \chi d y d x d t+\int_{\Omega} \int_{Y} \theta\left(f_{n}(0)\right) \chi d y d x .
\end{aligned}
$$

INRIA 
Since $\theta$ and $\theta^{\prime}$ are nonnegative and

$$
\theta^{*}\left(\theta^{\prime}(u)\right) \leq u \theta^{\prime}(u)-\theta(u) \leq C_{\theta} \theta(u), \quad u \geq 0,
$$

the Young inequality entails that

$$
\begin{aligned}
\int_{\Omega_{T}} \int_{Y_{\delta, R}} \theta^{\prime}\left(f_{n}\right) Q_{2}\left(f_{n}\right) d y d x d t \leq & \int_{\Omega_{T}} \int_{Y} \theta^{\prime}\left(f_{n}\right) Q_{2}\left(f_{n}\right) \chi d y d x d t \\
\leq & \int_{0}^{T} \int_{B_{2 R}} \int_{Y_{2 R}}\left\{\theta^{*}\left(\theta^{\prime}\left(f_{n}\right)\right)+\theta\left(Q_{1}\left(f_{n}\right)\right)\right\} d y d x d t \\
& +\frac{2}{\delta} \int_{0}^{T} \int_{B_{2 R}} \int_{Y_{\delta / 2,2 R}} \theta\left(f_{n}\right) p \cdot \nabla_{x} \chi d y d x d t+K_{1} \\
\leq & 2 K_{1}+\left(C_{\theta}+\frac{4 R}{\delta}\left\|\nabla_{x} \chi\right\|_{L^{\infty}}\right) \int_{0}^{T} \int_{B_{2 R}} \int_{Y_{2 R}} \theta\left(f_{n}\right) d y d x d t,
\end{aligned}
$$

whence (4.43).

Lemma 4.9 For each $R, T>0$, the sequence $\left(Q_{2}\left(f_{n}\right)\right)$ is weakly compact in $L^{1}((0, T) \times$ $\left.B_{R} \times Y_{R}\right)$.

Proof. We fix $T>0$ and $R>0$. Let $E$ be a measurable subset of $(0, T) \times B_{R} \times Y_{R}$ and $M, N, \delta, R_{1}$ and $R_{2}$ be positive real numbers such that $R_{1} \geq R$ and $R_{2} \geq 2 R_{1}^{2} / \delta$. Putting $\varphi=\mathbf{1}_{E}$ and

$$
A=A_{n}\left(t, x, y, y^{\prime}\right):=a\left(y, y^{\prime}\right) f_{n}(t, x, y) f_{n}\left(t, x, y^{\prime}\right),
$$

we have

$$
\int_{Y_{R}} Q_{2}\left(f_{n}\right) \varphi d y=\mathcal{I}_{2}+\mathcal{J}_{2}
$$

with

$$
\begin{aligned}
\mathcal{I}_{2}= & \int_{0}^{\delta} \int_{B_{R}} \int_{0}^{\infty} \int_{\mathbb{R}^{3}} A \varphi d y^{\prime} d y+\int_{Y_{\delta, R}} \int_{0}^{\delta} \int_{\mathbb{R}^{3}} A \varphi d y^{\prime} d y \\
& +\int_{Y_{\delta, R}} \int_{R_{1}}^{\infty} \int_{\mathbb{R}^{3}} A \varphi d y^{\prime} d y+\int_{Y_{\delta, R}} \int_{\delta}^{R_{1}} \int_{\left\{\left|p^{\prime}\right|>R_{2}\right\}} A \varphi d y^{\prime} d y \\
& +\int_{Y_{\delta, R}} \int_{\delta}^{R_{1}} \int_{B_{R_{2}}} A \varphi \mathbf{1}_{a \geq N} d y^{\prime} d y
\end{aligned}
$$

and

$$
\mathcal{J}_{2}=\int_{Y_{\delta, R}} \int_{\delta}^{R_{1}} \int_{B_{R_{2}}} A \varphi \mathbf{1}_{a \leq N} d y^{\prime} d y
$$


On the one hand, observe again that

$$
\frac{|p|}{m} \leq \frac{R}{\delta} \leq \frac{R_{2}}{2 R_{1}} \leq \frac{|p|+\left|p^{\prime}\right|}{m+m^{\prime}}
$$

so that

$$
m \Lambda\left(\frac{|p|}{m}, \frac{|p|+\left|p^{\prime}\right|}{m+m^{\prime}}\right) \geq \Lambda_{\delta, R}\left(R R_{2} / R_{1}\right):=\delta \Lambda\left(\frac{R}{\delta}, \frac{R_{2}}{2 R_{1}}\right)
$$

for $y \in Y_{\delta, R}$ and $y^{\prime} \in\left(\delta, R_{1}\right) \times B_{R_{2}}^{c}$, and that

$$
A=A\left(\mathbf{1}_{f_{n} \geq M}+\mathbf{1}_{f_{n}<M}\right) \leq A \frac{\theta^{\prime}\left(f_{n}\right)}{\theta^{\prime}(M)}+M \frac{a\left(y, y^{\prime}\right)}{\left|y^{\prime}\right|} f_{n}^{\prime}\left|y^{\prime}\right|
$$

on $Y_{\delta, R} \times\left(R_{1}, \infty\right) \times \mathbb{R}^{3}$. Using the growth conditions (1.9) and (1.10), a similar computation to the one performed to estimate $\mathcal{I}_{1}$ leads to

$$
\begin{aligned}
\mathcal{I}_{2} \leq & \frac{2}{r(\delta)} \mathcal{Y}_{r}\left(f_{n}(t, x)\right)+\frac{1}{\Lambda_{\delta, R}\left(R R_{2} / R_{1}\right)} \mathcal{E}_{\lambda}\left(f_{n}(t, x)\right) \\
& +\frac{1}{\theta^{\prime}(M)} \int_{Y_{\delta, R}} \theta^{\prime}\left(f_{n}\right) Q_{2}\left(f_{n}\right) d y+M \omega_{\delta, R}\left(R_{1}\right) \int_{Y}|y| f_{n}(t, x) d y \\
& +2 M a_{\delta, R_{2}}(N) \int_{Y} f_{n} d y+2 \frac{M}{\Phi(M)} D_{\Phi}^{1}\left(f_{n}(t, x)\right) .
\end{aligned}
$$

On the other hand, performing exactly the same computations as for $\mathcal{J}_{1}$, we obtain

$$
\mathcal{J}_{2} \leq 2 M N \int_{Y^{2}} f_{n} \varphi^{\prime} d y^{\prime} d y+\frac{2 M}{\Phi(M)} D_{\Phi}^{1}\left(f_{n}(t, x)\right) .
$$

We integrate (4.44) over $(0, T) \times B_{R}$ and use the estimates for $\mathcal{I}_{2}$ and $\mathcal{J}_{2}$ and Lemma 4.8 to obtain, thanks to (2.35) and (2.36),

$$
\begin{aligned}
\int_{0}^{T} \int_{B_{R}} \int_{Y_{R}} Q_{2}\left(f_{n}\right) \varphi d y d x d t \leq & \frac{2 K_{0}}{r(\delta)}+\frac{K(\delta, R)}{\theta^{\prime}(M)}+M \omega_{\delta, R}\left(R_{1}\right) K_{0} \\
& +\frac{K_{0}}{\Lambda_{\delta, R}\left(R R_{2} / R_{1}\right)}+2 M a_{\delta, R_{2}}(N) K_{0}+\frac{4 M}{\Phi(M)} K_{0} \\
& +2 M N \int_{0}^{T} \int_{B_{R}} \int_{Y^{2}} f_{n} \varphi^{\prime} d y^{\prime} d y d x d t .
\end{aligned}
$$

Using the already established weak compactness of $\left(f_{n}\right)$ in $L^{1}\left((0, T) \times B_{R} \times Y\right)$, we may pass to the limit first as $|E| \rightarrow 0$, then successively as $N \rightarrow+\infty, R_{2} \rightarrow+\infty$ and $R_{1} \rightarrow+\infty$, and finally as $M \rightarrow+\infty$ and $\delta \rightarrow 0$, to conclude.

Thanks to Lemma 4.7 and Lemma 4.9 there are a subsequence of $\left(f_{n}\right)$ (not relabeled) and nonnegative functions $f, \bar{Q}_{1}, \bar{Q}_{2}$ such that

$$
f_{n} \rightarrow f \quad \text { weakly in } \quad L^{1}\left((0, T) \times B_{R} \times Y\right),
$$


and

$$
Q_{i}\left(f_{n}\right) \rightarrow \bar{Q}_{i} \quad \text { weakly in } \quad L^{1}\left((0, T) \times B_{R} \times Y_{R}\right)
$$

for $i=1,2, T>0$ and $R>0$.

It remains to identify $\bar{Q}_{i}, i=1,2$, in terms of $f$. Since $Q\left(f_{n}\right)$ involves quadratic terms, some strong compactness is needed and is the subject of the next section.

\subsection{Strong compactness of $y$-averages}

Theorem 4.10 Let $T>0$ and consider two bounded sequences $\left(g_{n}\right)$ and $\left(G_{n}\right)$ of $L^{1}\left(\Omega_{T} \times Y\right)$ such that

$$
\partial_{t} g_{n}+v \cdot \nabla_{x} g_{n}=G_{n} \quad \text { in } \quad \Omega_{T} \times Y .
$$

Assume further that $\left(g_{n}\right)$ is weakly compact in $L^{1}\left((0, T) \times B_{R} \times Y_{R}\right)$ for each $R>0$. Then, for any $\psi=\psi\left(y, y^{\prime}\right) \in L^{\infty}\left(Y^{2}\right)$ with compact support, there holds

$$
\int_{Y} g_{n}(t, x, y) \psi\left(y, y^{\prime}\right) d y \quad \text { belongs to a strongly compact subset of } L^{1}\left((0, T) \times B_{R} \times Y_{R}\right)
$$

for each $R>0$.

This is a particular case of the velocity averaging lemma [7, 18, 29], noticing that $v=$ $v(y)=p / m$ satisfies the nondegeneracy condition

$$
\text { meas }\{y \in Y, \quad \sigma \cdot v(y)=u\}=0
$$

for every $\sigma \in \mathbb{S}^{2}$ and $u \in \mathbb{R}$. Nevertheless, it can be seen as a consequence of the classical averaging lemma [15, 18] as we show below.

Proof. First step. We assume further that $\left(g_{n}\right)$ is bounded in $L^{2}\left((0, T) \times B_{R} \times Y_{R}\right)$. We fix $\alpha=\alpha(m) \in C_{c}\left(\mathbb{R}_{+}\right)$and observe that

$$
\tilde{g}_{n}(t, x, v):=\int_{0}^{\infty} g_{n}(t, x, m, m v) \alpha(m) d m, \quad \tilde{G}_{n}(t, x, v):=\int_{0}^{\infty} G_{n}(t, x, m, m v) \alpha(m) d m
$$

belong respectively to a bounded subset of $L_{\mathrm{loc}}^{2}\left(\Omega_{T} \times \mathbb{R}^{3}\right)$ and $L_{\mathrm{loc}}^{1}\left(\Omega_{T} \times \mathbb{R}^{3}\right)$ and satisfies

$$
\frac{\partial \tilde{g}_{n}}{\partial t}+v \cdot \nabla_{x} \tilde{g}_{n}=\tilde{G}_{n} \quad \text { in } \quad(0, T) \times \Omega \times \mathbb{R}^{3} .
$$

Using the classical velocity averaging lemma [18, we get that

$$
\int_{\mathbb{R}^{3}} \int_{0}^{\infty} g_{n}(t, x, m, m v) \alpha(m) d m \beta(v) d v
$$

belongs to a strongly compact subset of $L_{\text {loc }}^{1}\left(\Omega_{T}\right)$ for every $\beta \in \mathcal{C}_{c}\left(\mathbb{R}^{3}\right)$. 
Escobedo, Laurençot \& Mischler

Second step. Performing the change of variables $(m, v) \rightarrow(m, p=m v)$, the previous step and the compactness of the support of $\alpha$ imply that 4.48 holds true with $\psi\left(y, y^{\prime}\right)=$ $\alpha(m) \beta(p / m) m^{-3}$.

We now fix $\chi \in \mathcal{C}_{c}\left(\mathbb{R}_{+}\right)$and $\zeta \in \mathcal{C}_{c}\left(\mathbb{R}^{3}\right)$. Consider $k \in \mathbb{N}$ and $\ell \in \mathbb{N}^{3}$. We deduce from the previous step with $\alpha(m)=m^{k} \chi(m)$ and $\beta(v)=v^{\ell} \zeta(v)$ that 4.48 is valid for $\psi\left(y, y^{\prime}\right)=m^{k-|\ell|-3} p^{\ell} \chi(m) \zeta(p / m)$. Therefore, (4.48) holds for $\psi\left(y, y^{\prime}\right)=\varphi(y) \chi(m) \zeta(p / m)$ for any $\varphi \in C_{c}(\bar{Y})$ by the Stone-Weierstrass theorem.

Choosing $\zeta \in \mathcal{C}_{c}\left(\mathbb{R}^{3}\right)$ such that $\zeta \equiv 1$ on $B_{\rho}$ for a sufficiently large $\rho$ (depending on the support of $\varphi$ and $\chi$ ), we realize that (4.48) is actually true for $\psi\left(y, y^{\prime}\right)=\varphi(y) \chi(m)$ for any $\varphi \in C_{c}(\bar{Y})$. Finally, using the weak compactness of $\left(g_{n}\right)$, we may let $\chi \rightarrow 1$ and deduce that (4.48) is valid for $\psi\left(y, y^{\prime}\right)=\varphi(y)$ for any $\varphi \in C_{c}(\bar{Y})$.

We then proceed as in [15, Corollary IV.2] to remove the additional assumption that $\left(g_{n}\right)$ is bounded in $L^{2}$ (derenormalization technique) and to extend the result to the class of functions $\psi \in L^{\infty}\left(Y^{2}\right)$ (density argument), thus completing the proof of Theorem 4.10]

\subsection{Passing to the limit in the coalescence term}

We aim to show that $\bar{Q}_{i}=Q_{i}(f)$ for $i=1,2$, where $f, \bar{Q}_{1}$ and $\bar{Q}_{2}$ are defined in (4.46) and (4.47.

Step 1. For $n \geq 1$, we put

$$
\rho_{n}:=\int_{Y} f_{n} d y, \quad \rho:=\int_{Y} f d y
$$

and claim that

$$
\rho_{n} \longrightarrow \rho \quad \text { in } \quad L^{1}\left((0, T) \times B_{R}\right) \quad \text { and a.e. in } \quad(0, T) \times B_{R}
$$

for each $R>0$. Indeed, fix $R>0$. We have $\rho_{n}=\rho_{n}^{M}+\sigma_{n}^{M}$ with

$$
\rho_{n}^{M}:=\int_{Y_{M}} f_{n} d y
$$

for $n \geq 1$ and $M>0$. By Theorem 4.10 $\left(\rho_{n}^{M}\right)$ belongs to a strongly compact subset of $L^{1}\left((0, T) \times B_{R}\right)$ for each $M>0$, while $\sigma_{n}^{M}$ satisfies

$$
\sigma_{n}^{M}:=\int_{Y_{M}^{c}} f_{n} d y \leq \frac{1}{M} \int_{Y} f_{n}|y| d y
$$

and thus converges to zero in $L^{1}\left((0, T) \times B_{R}\right)$ as $M \rightarrow+\infty$, the convergence being uniform with respect to $n \geq 1$ by (2.35). The claim (4.49) then readily follows.

Step 2. We next consider $\varphi \in \mathcal{D}(Y)$ and $R>0$ such that $\operatorname{supp} \varphi \subset Y_{R}$. Let $\delta, N, R_{1}$ and $R_{2}$ be positive real numbers such that $R_{1} \geq R+2 R^{2} / \delta$ and $R_{2} \geq 2 R_{1}^{2} / \delta$, and put

$$
b_{1}=\frac{1}{2} a \varphi^{\prime \prime} \mathbf{1}_{a \leq N} \mathbf{1}_{X_{1}}, \quad b_{2}=a \varphi \mathbf{1}_{a \leq N} \mathbf{1}_{X_{2}},
$$


with $X_{1}=\left\{\left(y, y^{\prime}\right) \in Y^{2} ; m, m^{\prime} \in[\delta, R], p^{\prime} \in B_{R_{1}}, p \in B_{R}\left(-p^{\prime}\right)\right\}, X_{2}=\left\{\left(y, y^{\prime}\right) \in Y^{2} ; y \in\right.$ $\left.Y_{\delta, R}, m^{\prime} \in\left[\delta, R_{1}\right], p^{\prime} \in B_{R_{2}}\right\}$.

We first claim that, for $i=1,2, \eta \in(0,1)$ and $R_{0}>0$, there holds

$$
\frac{1}{1+\eta \rho_{n}} \int_{Y} f_{n} \int_{Y} b_{i} f_{n}^{\prime} d y^{\prime} d y \rightarrow \frac{1}{1+\eta \rho} \int_{Y} f \int_{Y} b_{i} f^{\prime} d y^{\prime} d y
$$

weakly in $L^{1}\left((0, T) \times B_{R_{0}}\right)$. Indeed, notice that, since $b_{i} \in L^{\infty}\left(Y^{2}\right)$ with compact support, Theorem 4.10 and (4.49) imply that

$$
\frac{1}{1+\eta \rho_{n}} \int_{Y} b_{i} f_{n}^{\prime} d y^{\prime} \rightarrow \frac{1}{1+\eta \rho} \int_{Y} b_{i} f^{\prime} d y^{\prime} \quad \text { a.e. in } \quad(0, T) \times B_{R_{0}} \times Y_{R},
$$

and it is bounded in $L^{\infty}\left(\Omega_{T} \times Y\right)$ by $N\|\varphi\|_{L^{\infty}} / \eta$. Combining these properties with the weak convergence (4.46) of $\left(f_{n}\right)$ yields (4.50) by standard integration arguments, see [27, Lemma A.2] for instance.

We now aim at passing to the limit as $\eta \rightarrow 0$. As a first consequence of Lemma 4.9 and 4.50, we obtain that

$$
\begin{aligned}
\int_{0}^{T} \int_{B_{R_{0}}} \int_{Y^{2}} \frac{b_{i} f f^{\prime}}{1+\eta \rho} d y^{\prime} d y d x d t & \leq \liminf _{n \rightarrow+\infty} \int_{0}^{T} \int_{B_{R_{0}}} \int_{Y^{2}} \frac{b_{i} f_{n} f_{n}^{\prime}}{1+\eta \rho_{n}} d y^{\prime} d y d x d t \\
& \leq \sup _{n \geq 1} \int_{\Omega_{T}} \int_{Y} Q_{i}\left(f_{n}\right) d y d x d t
\end{aligned}
$$

for each $\eta \in(0,1)$. The parameters $R_{0}, R, N, \delta, R_{1}, R_{2}$ and $\eta$ being arbitrary, we deduce from the Fatou lemma that

$$
\int_{0}^{T} \int_{B_{R_{0}}} \int_{Y} \int_{Y} a \varphi f f^{\prime} d y^{\prime} d y d x d t \leq \sup _{n \geq 1} \int_{\Omega_{T}} \int_{Y} Q_{2}\left(f_{n}\right) d y d x d t<\infty .
$$

We next observe that, for $M \geq 1$ and $\eta \in(0,1)$, we have

$$
\frac{\eta \rho_{n}}{1+\eta \rho_{n}}=\frac{\eta \rho_{n}}{1+\eta \rho_{n}}\left(\mathbf{1}_{\rho_{n}<M}+\mathbf{1}_{\rho_{n} \geq M}\right) \leq \eta M+\mathbf{1}_{\rho_{n} \geq M} .
$$

Consequently,

$$
\begin{aligned}
\sup _{n \geq 1} \int_{0}^{T} \int_{B_{R_{0}}} \frac{\eta \rho_{n}}{1+\eta \rho_{n}} \int_{Y^{2}} b_{i} f_{n} f_{n}^{\prime} d y^{\prime} d y \leq & \eta M \sup _{n \geq 1} \int_{\Omega_{T}} \int_{Y_{R}} Q_{2}\left(f_{n}\right) d y d x d t \\
& +\sup _{n \geq 1} \int_{0}^{T} \int_{B_{R_{0}}} \int_{Y_{R}} \mathbf{1}_{\rho_{n} \geq M} Q_{2}\left(f_{n}\right) d y d x d t .
\end{aligned}
$$

We first use (2.36) to pass to the limit as $\eta \rightarrow 0$ and then use Lemma 4.9 and (4.49) to let $M \rightarrow+\infty$ and conclude that

$$
\lim _{\eta \rightarrow 0} \sup _{n \geq 1} \int_{0}^{T} \int_{B_{R_{0}}} \frac{\eta \rho_{n}}{1+\eta \rho_{n}} \int_{Y^{2}} b_{i} f_{n} f_{n}^{\prime} d y^{\prime} d y d x d t=0 .
$$

$\mathrm{RR} \mathrm{n}^{\circ} 4748$ 
Also, it follows from 4.50 that

$$
\lim _{\eta \rightarrow 0} \int_{0}^{T} \int_{B_{R_{0}}} \frac{\eta \rho}{1+\eta \rho} \int_{Y^{2}} b_{i} f f^{\prime} d y^{\prime} d y d x d t=0 .
$$

Combining the previous two identities with (4.50), we end up with

$$
\int_{Y} f_{n} \int_{Y} b_{i} f_{n}^{\prime} d y^{\prime} d y \rightarrow \int_{Y} f \int_{Y} b_{i} f^{\prime} d y^{\prime} d y
$$

weakly in $L^{1}\left((0, T) \times B_{R_{0}}\right)$.

Step 3. We now show that $\bar{Q}_{i}=Q_{i}(f)$ for $i=1,2$. For $i=1,2$, we write

$$
\int_{Y} Q_{i}\left(f_{n}\right) \varphi d y=\mathcal{I}_{i}^{n}+\int_{Y} f_{n} \int_{Y} b_{i} f_{n}^{\prime} d y^{\prime} d y,
$$

where $\mathcal{I}_{1}^{n}$ and $\mathcal{I}_{2}^{n}$ are given by (4.41) and 4.45) with $A=a f_{n} f_{n}^{\prime}$, respectively. Also,

$$
\int_{Y} Q_{i}(f) \varphi d y=\mathcal{I}_{i}+\int_{Y} f \int_{Y} b_{i} f^{\prime} d y^{\prime} d y
$$

where $\mathcal{I}_{1}$ and $\mathcal{I}_{2}$ are given by (4.41) and (4.45) with $A=a f f^{\prime}$, respectively. By (4.51) and the analysis of Section 3, we have

$$
\mathcal{I}_{i}^{n}+\mathcal{I}_{i} \rightarrow 0 \quad \text { in } \quad L^{1}\left(\Omega_{T} \times Y\right)
$$

uniformly with respect to $n \geq 1$ as $N \rightarrow+\infty, R_{2} \rightarrow+\infty, R_{1} \rightarrow+\infty$ and $\delta \rightarrow 0$. This last fact and 4.52) then imply that

$$
\int_{Y} Q_{i}\left(f_{n}\right) \varphi d y \rightarrow \int_{Y} Q_{i}(f) \varphi d y
$$

weakly in $L^{1}\left((0, T) \times B_{R_{0}}\right)$, from which we conclude that $\bar{Q}_{i}=Q_{i}(f)$ for $i=1,2$. Owing to (4.46) and (4.47), it is now a standard matter to pass to the limit in the equation satisfied by $f_{n}$ and obtain that $f$ satisfies (1.1) in the sense of distributions.

\subsection{Further properties of $f$}

In fact, owing to (2.35), (3.37) and the Dunford-Pettis theorem, the first assertion of Lemma 4.7 can be strenghtened.

Lemma 4.11 For $T>0$ and $R>0$, there is a weakly compact subset $\mathcal{K}_{R}$ of $L^{1}\left(B_{R} \times Y\right)$ such that $f_{n}(t) \in \mathcal{K}_{R}$ for each $t \in[0, T]$ and $n \geq 1$. 
We now improve the convergence (4.46) of $\left(f_{n}\right)$ to (3.39). Consider $T>0$ and $R>0$. On the one hand, it follows from Lemma 4.11 that $\left\{f_{n}(t), n \geq 1\right\}$ belongs to a weakly compact subset of $L^{1}\left(B_{R} \times Y\right)$ for each $t \in[0, T]$. On the other hand, we infer from (1.1) and (2.36) that

$$
\left|\int_{\Omega} \int_{Y}\left(f_{n}(t+h)-f_{n}(t)\right) \psi d y d x\right| \leq C(\psi) K_{0} h
$$

for every every $\psi \in \mathcal{D}(\Omega \times \bar{Y}), t \in[0, T)$ and $h \in(0, T-t)$, whence

$$
\lim _{h \rightarrow 0} \sup _{n \geq 1}\left|\int_{\Omega} \int_{Y}\left(f_{n}(t+h)-f_{n}(t)\right) \psi d y d x\right|=0
$$

for every $\psi \in \mathcal{D}(\Omega \times \bar{Y})$ and $t \in[0, T)$. A density argument and Lemma 4.7] then imply that (4.53) actually holds true for every $\psi \in L^{\infty}\left(B_{R} \times Y\right)$ (recall that a function in $L^{\infty}\left(B_{R} \times Y\right)$ is the pointwise limit of a sequence of functions in $\mathcal{D}(\Omega \times \bar{Y})$ which is bounded in $\left.L^{\infty}\left(B_{R} \times Y\right)\right)$. Therefore, a variant of the Arzelà-Ascoli theorem entails that, up to the extraction of a subsequence,

$$
f_{n} \longrightarrow f \quad \text { in } \quad \mathcal{C}\left([0, T] ; w-L^{1}\left(B_{R} \times Y\right)\right) .
$$

We next check that $f$ enjoys the properties listed in Definition 3.5 Owing to (4.46) and the convexity of $\Phi$, standard weak compactness arguments entail that $f$ satisfies (2.35). Next, proceeding as in Section 4.3, we obtain that $\mathcal{Y}_{1}(f), \mathcal{Y}_{r}(f)$ and $\mathcal{E}_{\lambda}(f)$ belong to $L^{1}\left(\mathbb{R}_{+} \times \Omega\right)$ with the help of the bound (2.36). It does not seem possible to use a similar argument to check that $D_{\Phi}(f)$ belongs to $L^{1}\left(\mathbb{R}_{+} \times \Omega\right)$ and we thus proceed directly on the equation satisfied by $f$. More precisely, we approximate $\Phi$ by a sequence of convex functions growing at most linearly at infinity and employ approximation arguments as in [16. Section 2] to show that $D_{\Phi}(f)$ belongs to $L^{1}\left(\mathbb{R}_{+} \times \Omega\right)$. We finally prove the strong continuity of $f$ as in [16. Corollary II.2] with the help of the $L^{1}$-bound on $r f$ to control the behaviour of $f$ for small $m$ and the following lemma to control the behaviour of $f$ for large $x$.

Lemma 4.12

$$
\lim _{R \rightarrow+\infty} \sup _{t \in[0, T]} \int_{\{|x| \geq R\}} \int_{Y} f(t, x, y) d y d x=0 .
$$

Proof. Let $A>0$. We multiply (1.1) by $m \wedge A$ and integrate over $Y$, which can be done, since $Q(f)$ and $f$ belong to $L^{1}\left(\Omega_{T} \times Y\right)$ for every $T>0$. The contribution of the collision term being nonnegative by $(2.19$, we get

$$
\partial_{t} \int_{Y}(m \wedge A) f(t, x, y) d y+\operatorname{div}_{x} \int_{Y}(m \wedge A) v f(t, x, y) d y \leq 0 \quad \text { in } \quad \mathcal{D}^{\prime}\left(\Omega_{T}\right) .
$$

We obtain (1.13) by passing to the limit $A \rightarrow+\infty$. 
Next, let $\xi \in \mathcal{C}^{\infty}\left(\mathbb{R}^{3}\right)$ be such that $0 \leq \xi \leq 1, \xi(x)=0$ if $|x| \leq 1 / 2$ and $\xi(x)=1$ if $|x| \geq 1$. For $R \geq 1$ and $x \in \mathbb{R}^{3}$, we put $\xi_{R}(x)=\xi(x / R)$. Let $R \geq 1$. We now multiply (1.13) by $\xi_{R}(x)$ and integrate over $\Omega_{t}$ to obtain

$$
\begin{aligned}
\int_{\Omega} \xi_{R}(x) \rho(t, x) d x & \leq \int_{\Omega} \xi_{R}(x) \rho^{i n}(x) d x+\frac{\|\nabla \xi\|_{L^{\infty}}}{R} \int_{0}^{t} \int_{\Omega} j(\tau, x) d x d \tau \\
& \leq \int_{\Omega} \xi_{R}(x) \rho^{i n}(x) d x+\frac{\|\nabla \xi\|_{L^{\infty}}}{R} K\left(f^{i n}\right) t
\end{aligned}
$$

thanks to (2.35).

Now, for $t \in[0, T]$ and $\delta \in(0,1)$, it follows from (2.35) and the above inequality that

$$
\begin{aligned}
\int_{\{|x| \geq R\}} \int_{Y} f(t, x, y) d y d x \leq & \frac{1}{r(\delta)} \int_{\{|x| \geq R\}} \int_{0}^{\delta} \int_{\mathbb{R}^{3}} r(m) f(t, x, y) d y d x \\
& +\frac{1}{\delta} \int_{\{|x| \geq R\}} \xi_{R}(x) \int_{\delta}^{\infty} \int_{\mathbb{R}^{3}} m f(t, x, y) d y d x \\
\leq & \frac{K\left(f^{i n}\right)}{r(\delta)}+\frac{1}{\delta} \int_{\{|x| \geq R / 2\}} \rho^{i n}(x) d x+\frac{\|\nabla \xi\|_{L^{\infty}}}{\delta R} K\left(f^{i n}\right) T .
\end{aligned}
$$

Lemma 4.12 then follows from (1.12) and (3.37) by letting first $R \rightarrow+\infty$ and then $\delta \rightarrow 0$ in the above inequality.

Let us finally briefly explain how it is possible to get the additional qualitative properties (1.14) - (1.16). The monotonicity (1.14) of the mass readily follows by integration of (1.13). We should actually have the mass conservation (2.20), at least for coagulation coefficients with a moderate growth, but we do not know how to prove it. For 1.15), we may consider a sequence $\left(\Phi_{k}\right)$ of convex functions growing at most linearly at infinity such that $\Phi_{k}(s) \nearrow s^{p}$ as $k \rightarrow \infty$ for any $s>0$ and we first establish that $\left\|\Phi_{k}(f(t))\right\|_{L^{1}}$ is a non-increasing function of time by a direct (and allowed) computation as it has been (formally) done in Lemma 2.3 We then let $k \rightarrow \infty$ and we conclude by the monotone convergence theorem. Concerning (1.16), we may argue as follows. We assume that the sequence of solutions $\left(f_{n}\right)$ satisfies

$$
t \longmapsto \int_{\Omega} \int_{Y} f_{n}(t, x, y) m \phi(m) \Psi(|v|) d y d x \text { is a non-increasing function of time, }
$$

for any decreasing function $\phi \in L^{\infty}\left(\mathbb{R}_{+}\right)$and any convex function $\Psi$ which grows at most quadratically at infinity. In particular, $\left\|f_{n}(t) m|v|^{2}\right\|_{L^{1}}$ is uniformly bounded. This is not a restrictive assumption, at least for an approximated solution, because (4.54) has been (formally) derived in Section 2. We then choose $\phi(m)=\phi_{R}(m)=\mathbf{1}_{0 \leq m \leq R}$ and $\Psi(s)=$ $\Psi_{R}(s)=s^{2} \mathbf{1}_{0 \leq s \leq R}+\left(2 R s-R^{2}\right) \mathbf{1}_{s \geq R}$. For $A \geq R$, we have

$$
\begin{gathered}
\int_{\Omega} \int_{Y} f_{n}(t, x, y) m \phi_{R}(m) \Psi_{R}(|v|) d y d x=\int_{\Omega} \int_{Y} f_{n}(t, x, y) m \phi_{R}(m) \Psi_{R}(|v|) \mathbf{1}_{|v| \leq A} d y d x \\
+\int_{\Omega} \int_{Y} f_{n}(t, x, y) m \phi_{R}(m) \Psi_{R}(|v|) \mathbf{1}_{|v|>A} d y d x .
\end{gathered}
$$


Since $m \phi_{R}(m) \Psi_{R}(|v|) \mathbf{1}_{|v| \leq A} \in L^{\infty}(Y)$ and

$$
\int_{\Omega} \int_{Y} f_{n}(t, x, y) m \phi_{R}(m) \Psi_{R}(|v|) \mathbf{1}_{|v|>A} d y d x \leq \frac{2 R}{A} \int_{\Omega} \int_{Y} f_{n}(t, x, y) m|v|^{2} d y d x \leq \frac{2 R}{A} C,
$$

we may pass to the limit as $n \rightarrow+\infty$ and deduce that

$$
\int_{\Omega} \int_{Y} f_{n}(t, x, y) m \phi_{R}(m) \Psi_{R}(|v|) d y d x \rightarrow \int_{\Omega} \int_{Y} f(t, x, y) m \phi_{R}(m) \Psi_{R}(|v|) d y d x .
$$

Therefore, recalling (4.54), we realize that

$$
t \longmapsto \int_{\Omega} \int_{Y} f(t, x, y) m \phi_{R}(m) \Psi_{R}(|v|) d y d x \quad \text { is a non-increasing function of time }
$$

for any $R \geq 0$. The assertion (1.16) then follows from [4.55), letting $R \rightarrow \infty$ and using the monotone convergence theorem.

\section{$5 \quad$ Large time behaviour and pressureless gases system}

We first prove the last assertion of Theorem 1.1. Let $f$ be a weak solution to (1.1)-(1.2) in the sense of Definition 3.5. We consider an increasing sequence $\left(t_{n}\right)_{n \geq 1}, t_{n} \rightarrow+\infty$, of positive real numbers and put $f_{n}(t,):.=f\left(t_{n}+t,.\right)$ for $t \geq 0$ and $n \geq 1$. Owing to Definition 3.5] we realize that the sequence $\left(f_{n}\right)$ satisfies the assumptions of Theorem [3.6 Therefore, fixing $T>0$, it follows from Theorem 3.6 that, up to the extraction of a subsequence,

$$
f_{n}(t) \rightarrow F \quad \text { weakly in } L^{1}\left(\Omega_{T} \times Y\right) .
$$

Moreover, on the one hand, we infer from (4.52) with $b_{2}=a \mathbf{1}_{a \leq N} \mathbf{1}_{Y_{\delta, R}^{2}}$ that

$$
\int_{Y_{\delta, R}^{2}} a \mathbf{1}_{a \leq N} f_{n} f_{n}^{\prime} d y^{\prime} d y \rightarrow \int_{Y_{\delta, R}^{2}} a \mathbf{1}_{a \leq N} F F^{\prime} d y^{\prime} d y \quad \text { weakly in } \quad L^{1}\left((0, T) \times B_{R}\right) .
$$

On the other hand, the bound (2.36) yields

$$
\int_{\Omega_{T}} \int_{Y^{2}} a f_{n} f_{n}^{\prime} d y^{\prime} d y d x d t \leq \int_{t_{n}}^{\infty} \int_{\Omega} \mathcal{Y}_{1}(f) d x d t \longrightarrow 0
$$

Therefore,

$$
\int_{0}^{T} \int_{B_{R}} \int_{Y_{\delta, R}^{2}} a \mathbf{1}_{a \leq N} F F^{\prime} d y^{\prime} d y d x d t=0
$$

whence

$$
a F F^{\prime}=0 \quad \text { a.e. on } \quad(0, T) \times \mathbb{R}^{3} \times Y^{2} .
$$

Consequently, $F \equiv 0$ a.e., since $0 \leq F \in L^{1}$ and $a>0$ a.e. on $Y^{2}$ by (1.7). 
Remark 5.13 When $\Omega=\mathbb{R}^{3}$ and $|x| f^{\text {in }} \in L^{1}(\Omega \times Y)$, an alternative proof of the convergence to zero may be performed with the help of [2.29) and (2.30). We refer to [4, 32] for more details about this dispersion argument.

Remark 5.14 It is mathematically challenging to have a better understanding of the relationship between the coalescence equation (1.1) and the pressureless gases system (1.18). Since colliding particles always stick together in the pressureless gases system, one can expect that it is the natural hydrodynamic limit of the coalescence equation:

$$
\partial_{t} f_{\varepsilon}+v \cdot \nabla_{x} f_{\varepsilon}=\frac{1}{\varepsilon} Q\left(f_{\varepsilon}\right) .
$$

This is not true if $f_{\varepsilon}(0)=f^{\text {in }}$ satisfies (1.12). Indeed, for $i=1,2$, we have

$$
\frac{1}{\varepsilon} \int_{\Omega_{T}} \int_{Y} Q_{i}\left(f_{\varepsilon}\right) d y d x d t \leq \frac{2}{\varepsilon} \int_{\Omega_{T}} \mathcal{Y}_{1}\left(f_{\varepsilon}\right) d x d t \leq 4\left\|f^{i n}\right\|_{L^{1}},
$$

and $\left\|\Phi\left(f_{\varepsilon}(t)\right)\right\|_{L^{1}} \leq\left\|\Phi\left(f^{i n}\right)\right\|_{L^{1}}$ for some $\Phi$ as in Definition 3.5. We may then argue as above to prove that $f_{\varepsilon} \rightarrow 0$ as $\varepsilon \rightarrow 0$. It would be interesting to figure out whether, if we start from a sequence of "well-prepared" initial data, the sequence $\left(f_{\varepsilon}\right)$ converges to a non-trivial solution to (1.1) of the form (1.17).

\section{Proof of the existence Theorem 1.1}

The proof is essentially the same as that in [27, Section 5] and proceeds in two steps: we first consider an approximated problem and then to use the stability result (Theorem 3.6) in order to pass to the limit. For the sake of completeness, we sketch below the main ingredients of these two steps.

Step 1. Approximation. We introduce the modified coalescence term

$$
Q_{\delta, \lambda}(g):=\tilde{Q}_{\delta}(g)+\lambda g
$$

with

$$
\tilde{Q}_{\delta}(g):=\frac{1}{1+\delta \rho_{g}} Q_{a_{\delta}}(g), \quad \rho_{g}:=\int_{Y}|g| d y
$$

and where $Q_{a_{\delta}}$ is defined by (1.3), (1.4) with the coalescence kernel $a_{\delta}\left(y, y^{\prime}\right):=\min \left\{a\left(y, y^{\prime}\right), 1 / \delta\right\}$.

For $\lambda \geq \delta^{-2}$ we obviously get that $Q_{\delta, \lambda}$ maps $X_{+}$in $X_{+}$, where the Banach space $\left(X,\|\cdot\|_{X}\right)$ is defined by

$$
X:=\left\{g \in L^{\infty}(Y) ; \quad(1+|y|)^{5} g \in L^{\infty}(Y)\right\},
$$

with $\|g\|_{X}:=\left\|(1+|y|)^{5} g\right\|_{L^{\infty}}$ and $X_{+}$denotes the positive cone of $X$. Moreover,

$$
\begin{aligned}
& \forall g \in X, \quad\left\|Q_{\delta, \lambda}(g)\right\|_{X} \leq C\|g\|_{X} \\
& \forall g_{1}, g_{2} \in X, \quad\left\|g_{i}\right\|_{X} \leq R \quad \Longrightarrow \quad\left\|Q_{\delta, \lambda}\left(g_{2}\right)-Q_{\delta, \lambda}\left(g_{1}\right)\right\|_{L^{1}(Y)} \leq C_{R}\left\|g_{2}-g_{1}\right\|_{L}(6.56)
\end{aligned}
$$

INRIA 
for some constants depending only on $\delta, \lambda$ (and $R$ ).

We next consider $f^{i n} \in L^{\infty}\left(\Omega ; X_{+}\right)$and $T>0$. Putting $B:=\left\|f^{i n}\right\|_{L^{\infty}(\Omega ; X)}$, we define the metric space $\left(X_{T}, d\right)$ by

$$
\begin{gathered}
X_{T}:=\left\{g \in \mathcal{C}\left([0, T] ; L^{1}(\Omega \times Y)\right) \cap L^{\infty}((0, T) \times \Omega ; X), \quad \sup _{t \in[0, T]}\|g(t)\|_{L^{\infty}(\Omega ; X)} \leq 2 B\right\}, \\
d\left(g_{1}, g_{2}\right):=\sup _{t \in[0, T]}\left\|\left(g_{1}-g_{2}\right)(t)\right\|_{L^{1}(\Omega \times Y)}, \quad g_{1}, g_{2} \in X_{T},
\end{gathered}
$$

and the map $\Phi: X_{T} \rightarrow X_{T}$ in the following way: for any $g \in X_{T}, h=\Phi(g)$ is the unique solution to

$$
\partial_{t} h+v \cdot \nabla_{x} h+\lambda h=Q_{\delta, \lambda}(g), \quad h(0)=f^{i n},
$$

with periodic boundary conditions when $\Omega$ is the torus and with the noincoming-flux boundary conditions when $\Omega$ is bounded. It is straightforward to verify that $\Phi$ is well-defined and satisfies

$$
g \geq 0 \quad \Longrightarrow \quad \Phi(g) \geq 0,
$$

by the maximum principle for $\lambda>\delta^{-2}$. In addition, we infer from (6.56) and (6.57) that there exist $T>0$ and $k \in(0,1)$ such that

$$
g \in X_{T} \quad \Longrightarrow \quad \Phi(g) \in X_{T}
$$

and

$$
d\left(\Phi\left(g_{1}\right), \Phi\left(g_{2}\right)\right) \leq k d\left(g_{1}, g_{2}\right) \quad \text { for } \quad g_{1}, g_{2} \in X_{T} .
$$

By the contracting map theorem, $\Phi$ has a unique fixed point $f_{\delta}$ in $X_{T}$, which is actually a solution to the modified coalescence equation

$$
\begin{aligned}
\partial_{t} f_{\delta}+v \cdot \nabla_{x} f_{\delta} & =\tilde{Q}_{\delta}\left(f_{\delta}\right) \text { in }(0, T) \times \Omega \times Y, \\
f_{\delta}(0) & =f^{i n} \text { in } \Omega \times Y,
\end{aligned}
$$

with periodic boundary conditions when $\Omega$ is the torus and with the noincoming-flux boundary conditions when $\Omega$ is bounded.

By standard iterative arguments, (6.56) ensures that $f_{\delta}$ is in fact a global solution (with $T=\infty)$ and satisfies

$$
\left\|f_{\delta}(t)\right\|_{L^{\infty}(\Omega ; X)} \leq \exp (C(1+t)), \quad t \geq 0 .
$$

Step 2. The limit $\delta \rightarrow 0$. Given now an initial data $f^{\text {in }}$ satisfying the condition (1.12), we introduce the approximation $f_{\delta}^{i n}$ given by

$$
f_{\delta}^{i n}(x, y):=\min \left\{f^{i n}(x, y), \frac{1}{\delta}\right\} \mathbf{1}_{\Omega_{\delta}}(x) \mathbf{1}_{B_{1 / \delta}}(y)
$$

$\mathrm{RR} \mathrm{n}^{\circ} 4748$ 
with $\Omega_{\delta}=\Omega$ when $\Omega$ is either the torus or a bounded domain, and $\Omega_{\delta}:=B_{1 / \delta}$ when $\Omega=\mathbb{R}^{3}$. Clearly, $f_{\delta}^{i n} \in X_{+}$and we denote by $f_{\delta}$ the corresponding solution to the modified coalescence equation (6.58), 6.59).

We first show that, under the assumption (1.12), the initial data $f^{i n}$ and $f_{\delta}^{i n}$ satisfy (2.34) uniformly with respect to $\delta$. For that purpose, we collect some properties of $f^{i n}$ in the next lemma.

Lemma 6.15 Assume that $f^{\text {in }}$ satisfies (1.12). There exist two nonnegative and nondecreasing convex functions $\Phi \in \mathcal{C}^{1}([0,+\infty))$ and $\lambda \in \mathcal{C}^{1}([0,+\infty))$ and a nonnegative, convex and non-increasing function $r \in \mathcal{C}^{1}((0,+\infty))$ depending only on $f^{\text {in }}$ satisfying $\Phi(0)=0$, $\lambda(0)=0$, (3.37) and such that

$$
\int_{\Omega} \int_{Y} \Phi\left(f^{i n}(x, y)\right)+f^{i n}(x, y)\left(r(m)+\lambda\left(\frac{|p|}{m}\right)\right) d y d x<+\infty .
$$

Proof. Since $f^{i n} \in L^{1}(\Omega \times Y)$, the de la Vallée-Poussin theorem [14, 33] ensures that there is a nonnegative and non-decreasing convex function $\Phi \in \mathcal{C}^{1}([0,+\infty))$ satisfying $\Phi(0)=0$, (3.37) and $\Phi\left(f^{i n}\right) \in L^{1}(\Omega \times Y)$. Similarly, it follows from (1.12) that $(x, y) \mapsto p / m$ belongs to $L^{1}\left(\Omega \times Y ; m f^{i n}(x, y) d x d y\right)$ and the de la Vallée-Poussin theorem [14, 33] ensures that there is a nonnegative and non-decreasing convex function $\lambda \in \mathcal{C}^{1}([0,+\infty))$ satisfying $\lambda(0)=0$, 3.37 and

$$
\int_{\Omega} \int_{Y} m \lambda\left(\frac{|p|}{m}\right) f^{i n}(x, y) d y d x<+\infty .
$$

We next notice that $f^{i n} \in L^{1}(\Omega \times Y)$ implies that

$$
(x, y) \longmapsto \frac{1}{m} \in L^{1}\left(\Omega \times Y ; m f^{i n}(x, y) d x d y\right) .
$$

Using again the de la Vallée-Poussin theorem, we conclude that there is a nonnegative, non-increasing and convex function $\sigma \in \mathcal{C}^{1}([0,+\infty))$ satisfying $\sigma(0)=0, \sigma(u) / u \rightarrow+\infty$ as $u \rightarrow+\infty$ and

$$
(x, y) \longmapsto \sigma\left(\frac{1}{m}\right) \in L^{1}\left(\Omega \times Y ; m f^{i n}(x, y) d x d y\right) .
$$

Putting $r(m):=m \sigma(1 / m)$ for $m>0$, this last fact implies that $f^{i n} \in L^{1}(\Omega \times Y, r(m) d x d y)$, while the behaviour of $\sigma$ for large $u$ entails that $r$ fulfils (3.37). Next, since $\sigma$ is a convex function with $\sigma(0)=0, m \mapsto \sigma(m) / m$ is a non-decreasing function and $r$ is thus a nonincreasing function. The other properties of $r$ finally follows from the properties of $\sigma$. Summarizing, we have constructed three functions $\Phi, \lambda$ and $r$ with the properties stated in Lemma 6.15] such that 6.60 holds true.

Since $f_{\delta}^{i n} \leq f^{i n}$, we readily obtain that (6.60) holds for $\left(f_{\delta}^{i n}\right)$ uniformly with respect to $\delta$. Owing to the properties of $f_{\delta}$, we may justify the computations performed in Section 2 and deduce that the family $\left\{f_{\delta}\right\}$ satisfies the bounds (2.35) and (2.36) uniformly with respect 
to $\delta$ (notice also that the modified coalescence kernel $a_{\delta} /\left(1+\delta \rho_{f_{\delta}}\right)$ satisfies (1.7)-(1.10) uniformly with respect to $\delta$ ). Arguing as in the proof of Theorem [3.6] in the same way as it is done in [27, we deduce that there are a subsequence $\left(f_{\delta_{k}}\right)$ of $\left(f_{\delta}\right)$ and a function $f$ such that

$$
\left\{\begin{array}{l}
f_{\delta_{k}} \longrightarrow f \text { in } \mathcal{C}\left([0, T) ; w-L^{1}\left(B_{R} \times Y\right)\right), \\
\tilde{Q}_{\delta_{k}}\left(f_{\delta_{k}}\right) \rightarrow Q(f) \text { in } L^{1}\left((0, T) \times B_{R} \times Y_{R}\right)
\end{array}\right.
$$

for any $T>0$ and $R>0$. We therefore conclude that $f$ is a weak solution to (1.1)-(1.2) enjoying the properties stated in Theorem 1.1

\section{A From $(r, v)$ to $(m, p)$.}

We first show in this appendix that the equation (1.1) with the collision term $Q(f)$ given by (1.3) and (1.4) is deduced from the usual droplet equation as it is presented for instance in [36, through a simple change of variables.

Let us consider a function $g=g(t, x, r, v)$ which solves the equation:

$$
\partial_{t} g+v \cdot \nabla_{x} g=\mathcal{Q}(g) \quad \text { in } \quad(0,+\infty) \times \Omega \times \mathbb{R}_{+} \times \mathbb{R}^{3},
$$

where $\mathcal{Q}(g):=\mathcal{Q}_{1}(g)-\mathcal{Q}_{2}(g)$ is given by

$$
\begin{aligned}
& \mathcal{Q}_{1}(g)(r, v)=\frac{1}{2} \int_{\mathbb{R}^{3}} \int_{0}^{r} \frac{r^{11}}{r_{1}^{11}} B\left(r_{1}, r^{*}, v_{1}, v^{*}\right) g\left(r_{1}, v_{1}\right) g\left(r^{*}, v^{*}\right) d r^{*} d v^{*}, \\
& \mathcal{Q}_{2}(g)(r, v)=\int_{\mathbb{R}^{3}} \int_{0}^{\infty} B\left(r, r^{*}, v, v^{*}\right) g(r, v) g\left(r^{*}, v^{*}\right) d r^{*} d v^{*},
\end{aligned}
$$

and

$$
\begin{aligned}
& r_{1}=\left(r^{3}-r^{* 3}\right)^{1 / 3}, \quad v_{1}=\frac{r^{3} v-r^{* 3} v^{*}}{r^{3}-r^{* 3}}, \\
& B\left(r, r^{*}, v, v^{*}\right)=E\left(r, r^{*}, v, v^{*}\right) \pi\left(r+r^{*}\right)^{2}\left|v-v^{*}\right|,
\end{aligned}
$$

the function $E$ being a positive function, called the coalescence efficiency. We perform the following change of variables:

$$
m:=r^{3}, p:=r^{3} v, \text { and } \quad f(t, x, m, p):=\frac{m^{-11 / 3}}{3} g\left(t, x, m^{1 / 3}, \frac{p}{m}\right) .
$$

It then follows from that

$$
\begin{aligned}
\partial_{t} f(t, x, m, p) & =\frac{m^{-11 / 3}}{3} \partial_{t} g\left(t, x, m^{1 / 3}, \frac{p}{m}\right) \\
& =\frac{m^{-11 / 3}}{3} \mathcal{Q}_{1}(g)\left(t, x, m^{1 / 3}, \frac{p}{m}\right)-\frac{m^{-11 / 3}}{3} \mathcal{Q}_{2}(g)\left(t, x, m^{1 / 3}, \frac{p}{m}\right) .
\end{aligned}
$$

$\mathrm{RR} \mathrm{n}^{\circ} 4748$ 
We now compute the two terms on the right-hand side. Starting with $\mathcal{Q}_{2}$, we obtain $\mathcal{Q}_{2}(g)\left(t, x, m^{1 / 3}, \frac{p}{m}\right)=g\left(t, x, m^{1 / 3}, \frac{p}{m}\right) \int_{\mathbb{R}^{3}} \int_{0}^{\infty} B\left(m^{1 / 3}, r^{*}, \frac{p}{m}, v^{*}\right) g\left(t, x, r^{*} v^{*}\right) d r^{*}, d v^{*}$.

We change variables under the integral

$$
m^{*}=r^{* 3}, \quad p^{*}=r^{* 3} v^{*}, \quad d r^{*} d v^{*}=\frac{1}{3} m^{*-11 / 3} d m^{*} d p^{*},
$$

and obtain,

$$
\mathcal{Q}_{2}(g)\left(t, x, m^{1 / 3}, \frac{p}{m}\right)=3 m^{11 / 3} f(t, x, m, p) \int_{\mathbb{R}^{3}} \int_{0}^{\infty} a\left(m, p, m^{*}, p^{*}\right) f\left(t, x, m^{*}, p^{*}\right) d m^{*} d p^{*}
$$

with

$$
a\left(m, p, m^{*}, p^{*}\right)=B\left(m^{1 / 3}, m^{* 1 / 3}, \frac{p}{m}, \frac{p^{*}}{m^{*}}\right) .
$$

On the other hand, using the same change of variables and A.64, we obtain

$$
\begin{aligned}
& \mathcal{Q}_{1}(g)\left(t, x, m^{1 / 3}, \frac{p}{m}\right) \\
= & \frac{1}{2} \int_{\mathbb{R}^{3}} \int_{0}^{m^{1 / 3}} \frac{m^{11 / 3}}{r_{1}^{11}} B\left(r_{1}, r^{*}, v_{1}, v^{*}\right) g\left(t, x, r_{1}, v_{1}\right) g\left(t, x, r^{*}, v^{*}\right) d r^{*} d v^{*} \\
= & \frac{3}{2} m^{11 / 3} \int_{\mathbb{R}^{3}} \int_{0}^{m} a\left(m-m^{*}, p-p^{*}, m^{*}, p^{*}\right) f\left(t, x, m-m^{*}, p-p^{*}\right) f\left(t, x, m^{*}, p^{*}\right) d m^{*} d p^{*} .
\end{aligned}
$$

Using the notation $y=(m, p)$ introduced in Section 1 and A.65, we may write $a$ as follows

$$
a\left(y, y^{\prime}\right)=B\left(m^{1 / 3},\left(m^{\prime}\right)^{1 / 3}, \frac{p}{m}, \frac{p^{\prime}}{m^{\prime}}\right)=E\left(m^{1 / 3},\left(m^{\prime}\right)^{1 / 3}, v, v^{\prime}\right)\left(m^{1 / 3}+\left(m^{\prime}\right)^{1 / 3}\right)^{2}\left|v-v^{\prime}\right| .
$$

\section{B Properties of $a_{H S}$ and $a_{N P}$}

We check here that Theorem 1.1 guarantees the existence of a weak solution to (1.1)-(1.2) when the coalescence kernels are given by either (1.5) or (1.6). For that purpose, we only have to show that these kernels enjoys the properties [1.8-1.10. We first consider $a_{H S}$ and prove the following result.

Lemma B.16 For any $\alpha \geq 0$ the function

$$
a\left(y, y^{\prime}\right)=\left(m^{\alpha}+m^{\prime \alpha}\right)^{2}\left|v-v^{\prime}\right|
$$

satisfies (1.8) and (1.9). Moreover, if $0 \leq \alpha<1 / 2$, it also satisfies (1.10). 
Proof. Let us start with (1.8). To this end, we notice that,

$$
\left|v-v^{\prime \prime}\right|=\left|\frac{v\left(m+m^{\prime}\right)}{m^{\prime \prime}}-\frac{m v+m^{\prime} v^{\prime}}{m^{\prime \prime}}\right|=\frac{m^{\prime}}{m^{\prime \prime}}\left|v-v^{\prime}\right|,
$$

and similarly,

$$
\left|v^{\prime}-v^{\prime \prime}\right|=\frac{m}{m^{\prime \prime}}\left|v-v^{\prime}\right|
$$

Therefore,

$$
\begin{aligned}
a\left(y, y^{\prime \prime}\right)+a\left(y^{\prime}, y^{\prime \prime}\right) & =\left\{\left(m^{\alpha}+m^{\prime \prime \alpha}\right)^{2} \frac{m^{\prime}}{m^{\prime \prime}}+\left(m^{\prime \alpha}+m^{\prime \prime \alpha}\right)^{2} \frac{m}{m^{\prime \prime}}\right\}\left|v-v^{\prime}\right| \\
& \geq\left\{\left(m^{\alpha}+m^{\prime \alpha}\right)^{2} \frac{m^{\prime}}{m^{\prime \prime}}+\left(m^{\prime \alpha}+m^{\alpha}\right)^{2} \frac{m}{m^{\prime \prime}}\right\}\left|v-v^{\prime}\right| \\
& \geq\left(m^{\alpha}+m^{\prime \alpha}\right)^{2}\left|v-v^{\prime}\right|=a\left(y, y^{\prime}\right) .
\end{aligned}
$$

On the other hand, it is clear that $a$ is bounded on $Y_{\delta, R}^{2}$, so that (1.9) is obviously true. Finally, if $y^{\prime} \in Y$ is such that $m^{\prime} \geq R^{\prime} \geq 1+R$ and $y \in Y_{\delta, R}$, we have

$$
\begin{aligned}
a\left(y, y^{\prime}\right) & \leq\left(R^{\alpha}+m^{\prime \alpha}\right)^{2}\left(\frac{R}{\delta}+\left|v^{\prime}\right|\right) \leq C(R, \delta)\left(m^{2 \alpha}+m^{2 \alpha-1}\left|p^{\prime}\right|\right) \\
& \leq \frac{C(R, \delta)}{R^{\prime 1-2 \alpha}}\left|y^{\prime}\right|
\end{aligned}
$$

and (1.10) follows since $\alpha \in[0,1 / 2)$.

Lemma B.17 For $\alpha \in[0,1]$ and $\gamma \in \mathbb{R}$, we put

$$
a\left(y, y^{\prime}\right)=\left(\frac{m+m^{\prime}}{m m^{\prime}}\right)^{\alpha}\left|v-v^{\prime}\right|^{\gamma} .
$$

If $-3<\gamma \leq 0$, the function a satisfies (1.8)-(1.10).

Proof. Since $\alpha \leq 1$, it follows from the formulae for $\left|v-v^{\prime \prime}\right|$ and $\left|v^{\prime}-v^{\prime \prime}\right|$ computed in the proof of Lemma B.16 and the subadditivity of $u \mapsto u^{\alpha}$ that

$$
\begin{aligned}
a\left(y, y^{\prime \prime}\right)+a\left(y^{\prime}, y^{\prime \prime}\right) & \geq\left\{\frac{m+m^{\prime \prime}}{m m^{\prime \prime}}\left(\frac{m^{\prime}}{m^{\prime \prime}}\right)^{\gamma / \alpha}+\frac{m^{\prime}+m^{\prime \prime}}{m^{\prime} m^{\prime \prime}}\left(\frac{m}{m^{\prime \prime}}\right)^{\gamma / \alpha}\right\}^{\alpha}\left|v-v^{\prime}\right|^{\gamma} \\
& \geq a\left(y, y^{\prime}\right)\left\{\left(\frac{m^{\prime}}{m^{\prime \prime}}\right)^{(\alpha+\gamma) / \alpha}+\left(\frac{m}{m^{\prime \prime}}\right)^{(\alpha+\gamma) / \alpha}\right\}^{\alpha} .
\end{aligned}
$$


Since $(\alpha+\gamma) / \alpha \leq 1$, the function $u \mapsto u^{(\alpha+\gamma) / \alpha}$ is subadditive, from which we deduce that

$$
a\left(y, y^{\prime \prime}\right)+a\left(y^{\prime}, y^{\prime \prime}\right) \geq a\left(y, y^{\prime}\right)\left\{\frac{m^{\prime}}{m^{\prime \prime}}+\frac{m}{m^{\prime \prime}}\right\}^{\gamma+\alpha}=a\left(y, y^{\prime}\right) .
$$

We next turn to (1.9). Since $\gamma>-3$, there exists $q>1$ such that $\gamma q>-3$. For $y^{\prime} \in Y_{\delta, R}$, we have

$$
\begin{aligned}
\int_{Y_{\delta, R}} a\left(y, y^{\prime}\right)^{q} d y & \leq\left(\frac{2}{\delta}\right)^{\alpha q} \int_{Y_{\delta, R}}\left|p-\frac{m p^{\prime}}{m^{\prime}}\right|^{\gamma q} m^{-\gamma q} d y \\
& \leq C R^{-\gamma q} \int_{\delta}^{R} \int_{B_{R}\left(-m p^{\prime} / m^{\prime}\right)}|p|^{\gamma q} d y \\
& \leq C \int_{B_{R+R^{2} / \delta}}|p|^{\gamma q} d p
\end{aligned}
$$

which is finite since $\gamma q>-3$ and does not depend on $y^{\prime} \in Y_{\delta, R}$. The property (1.9) then follows from the inequality

$$
\int_{Y_{\delta, R}} a\left(y, y^{\prime}\right) \mathbf{1}_{a\left(y, y^{\prime}\right) \geq M} d y \leq \frac{1}{M^{q-1}} \int_{Y_{\delta, R}} a\left(y, y^{\prime}\right)^{q} d y .
$$

Finally, we have

$$
\int_{Y_{\delta, R}} a\left(y, y^{\prime}\right) d y \leq\left(\frac{2}{\delta}\right)^{\alpha} \int_{Y_{\delta, R}}\left|\frac{p}{m}-\frac{p^{\prime}}{m^{\prime}}\right|^{\gamma} d y
$$

for $m^{\prime} \geq R^{\prime} \geq R$ and $p^{\prime} \in \mathbb{R}^{3}$. Now, if $\left|p^{\prime}\right| / m^{\prime} \geq 2 R / \delta$, we have

$$
\left|\frac{p}{m}-\frac{p^{\prime}}{m^{\prime}}\right| \geq \frac{\left|p^{\prime}\right|}{m^{\prime}}-\frac{|p|}{m} \geq \frac{\left|p^{\prime}\right|}{2 m^{\prime}}
$$

and

$$
\int_{Y_{\delta, R}} a\left(y, y^{\prime}\right) d y \leq C(\delta, R) \int_{Y_{\delta, R}}\left|\frac{p^{\prime}}{m^{\prime}}\right|^{\gamma} d y \leq C(\delta, R)
$$

since $\gamma \leq 0$. On the other hand, if $\left|p^{\prime}\right| / m^{\prime}<2 R / \delta$, we have $B_{R / m}\left(-p^{\prime} / m^{\prime}\right) \subset B_{3 R / \delta}$ and

$$
\int_{Y_{\delta, R}} a\left(y, y^{\prime}\right) d y \leq C(\delta, R) \int_{\delta}^{R} \int_{B_{R / m}\left(-p^{\prime} / m^{\prime}\right)} m^{3}|p|^{\gamma} d y \leq C(\delta, R)
$$

since $\gamma>-3$. The assertion (1.10) then readily follows. 


\section{References}

[1] D.J. Aldous, Deterministic and stochastic models for coalescence (aggregation, coagulation): a review of the mean-field theory for probabilists, Bernoulli 5 (1999), 3-48.

[2] H. Amann, Coagulation-fragmentation processes, Arch. Rational Mech. Anal. 151 (2000), 339-366.

[3] C. Baranger, Collisions, coalescences et fragmentations des gouttelettes dans un spray: écriture précise des équations relatives au modèle TAB, mémoire de DEA, ENS de Cachan, 2001.

[4] C. Bardos and P. Degond, Global existence for the Vlasov-Poisson equation in 3 space variables with small initial data, Ann. Inst. H. Poincaré Anal. Non Linéaire 2 (1985), 101-118.

[5] Ph. Bénilan and D. Wrzosek, On an infinite system of reaction-diffusion equations, Adv. Math. Sci. Appl. 7 (1997), 349-364.

[6] A.V. Bobylev and R. Illner, Collision integrals for attractive potentials, J. Statist. Phys. 95 (1999), 633-649.

[7] F. Bouchut, F. Golse and M. Pulvirenti, Kinetic equations and asymptotic theory, Series in Appl. Math., Gauthiers-Villars, 2000.

[8] F. Bouchut and F. James, Duality solutions for pressureless gases, monotone scalar conservation laws, and uniqueness, Comm. Partial Differential Equations 24 (1999), 2173-2189.

[9] Y. Brenier and E. Grenier, Sticky particles and scalar conservation laws, SIAM J. Numer. Anal. 35 (1998), 2317-2328.

[10] P.R. Brazier-Smith, S.G. Jennings and J. Latham, The interaction of falling water drops: coalescence, Proc. R. Soc. London 326 (1972), 393-408.

[11] J.F. Collet and F. Poupaud, Existence of solutions to coagulation-fragmentation systems with diffusion, Transport Theory Statist. Phys. 25 (1996), 503-513.

[12] M.H. Davies and J.D. Sartor, Theoretical collision efficiencies for small cloud droplets in Stokes flow, Nature 215 (1967), 1371-1372.

[13] M. Deaconu and N. Fournier, Probabilistic approach of some discrete and continuous coagulation equations with diffusion, Stochastic Process. Appl. 101 (2002), 83-111.

[14] C. Dellacherie and P.A. Meyer, Probabilités et potentiel, chapitres I à IV, Hermann, Paris, 1975.

[15] R.J. DiPerna and P.-L. Lions, On the Cauchy problem for Boltzmann equations : global existence and stability, Ann. Math. 130 (1989), 321-366.

[16] R.J. DiPerna and P.-L. Lions, Ordinary differential equations, transport theory and Sobolev spaces, Invent. Math. 98 (1989), 511-547.

[17] R.J. DiPerna and P.-L. Lions, Global solutions of Boltzmann's equation and the entropy inequality, Arch. Rational Mech. Anal. 114 (1991), 47-55.

[18] R.J. DiPerna, P.-L. Lions and Y. Meyer, $L^{p}$ regularity of velocity averages, Ann. Inst. H. Poincaré Anal. Non Linéaire 8 (1991), 271-287.

[19] R.L. Drake, A general mathematical survey of the coagulation equation, in "Topics in Current Aerosol Research (part 2)," International Reviews in Aerosol Physics and Chemistry, Pergamon Press, Oxford, 1972, pp. 203-376.

[20] P.B. Dubovskii, Mathematical Theory of Coagulation, Lecture Notes Series 23, Seoul National University, Research Institute of Mathematics, Global Analysis Research Center, Seoul, 1994.

[21] G. Dufour, M. Massot and Ph. Villedieu, Etude d'un modèle de fragmentation secondaire pour les brouillards de gouttelettes, preprint, 2002.

[22] S. K. Friedlander, Smoke, Dust and Haze, Addison-Wesley Publishing Company, 1977. 
[23] R. Illner, Stellar dynamics and plasma physics with corrected potentials: Vlasov, Manev, Boltzmann, Smoluchowski, Hydrodynamic limits and related topics (Toronto, ON, 1998), 95-108, Fields Inst. Commun., 27, Amer. Math. Soc., Providence, RI, 2000.

[24] P.E. Jabin and J. Soler, A kinetic description of particle fragmentations, preprint, 2002.

[25] I. Langmuir, The production of rain by a chain reaction in cumulus clouds at temperature above freezing, J. Meteor. 5 (1948) 175-192.

[26] Ph. Laurençot and S. Mischler, Global existence for the discrete diffusive coagulation-fragmentation equations in $L^{1}$, Rev. Mat. Iberoamericana 18 (2002), 221-235.

[27] Ph. Laurençot and S. Mischler, The continuous coagulation-fragmentation equations with diffusion, Arch. Rational Mech. Anal. 162 (2002), 45-99.

[28] Lê Châu-Hoàn, Etude de la classe des opérateurs m-accrétifs de $L^{1}(\Omega)$ et accrétifs dans $L^{\infty}(\Omega)$, Thèse de $3^{\text {ème }}$ cycle, Université de Paris VI, 1977.

[29] P.-L. Lions, B. Perthame and E. Tadmor, A kinetic formulation of multidimensional scalar conservation laws and related equations, J. Amer. Math. Soc. 7 (1994), 169-191.

[30] Z.A. Melzak, A scalar transport equation, Trans. Amer. Math. Soc. 85 (1957), 547-560.

[31] S. Mischler and M. Rodriguez Ricard, Existence globale pour l'équation de Smoluchowski continue non homogène et comportement asymptotique des solutions, prépublication Université de Versailles Saint-Quentin, 2002.

[32] B. Perthame, Time decay, propagation of low moments and dispersive effects for kinetic equations, Comm. Partial Differential Equations 21 (1996), 659-686.

[33] M.M. Rao and Z.D. Ren, Theory of Orlicz Spaces, Monogr. Textbooks Pure Appl. Math. 146, Marcel Dekker, Inc., New York, 1991.

[34] P.J. O'Rourke, Collective drop effects on vaporizing liquid sprays, Ph.D. Thesis, Los Alamos National Laboratory, Los Alamos, NM 87545, November 1981.

[35] J.M. Roquejoffre and Ph. Villedieu, A kinetic model for droplet coalescence in dense sprays, Math. Models Methods Appl. Sci. 11 (2001), 867-882.

[36] Ph. Villedieu and J. Hylkema, Une méthode particulaire aléatoire reposant sur une équation cinétique pour la simulation numérique des sprays denses de gouttelettes liquides, C. R. Acad. Sci. Paris Sér. I Math. 325 (1997), 323-328.

[37] F.A. Williams, Combustion theory, Addison-Wesley Publishing Company, 1985.

[38] Ya.B. Zeldovich, Gravitational instability: an approximate theory for large density perturbations, Astron. Astrophys. 5 (1970), 84-89. 


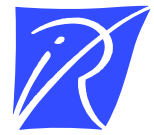

Unité de recherche INRIA Rocquencourt

Domaine de Voluceau - Rocquencourt - BP 105 - 78153 Le Chesnay Cedex (France)

Unité de recherche INRIA Futurs : Domaine de Voluceau - Rocquencourt - BP 105 - 78153 Le Chesnay Cedex (France) Unité de recherche INRIA Lorraine : LORIA, Technopôle de Nancy-Brabois - Campus scientifique 615, rue du Jardin Botanique - BP 101 - 54602 Villers-lès-Nancy Cedex (France)

Unité de recherche INRIA Rennes : IRISA, Campus universitaire de Beaulieu - 35042 Rennes Cedex (France)

Unité de recherche INRIA Rhône-Alpes : 655, avenue de l'Europe - 38330 Montbonnot-St-Martin (France)

Unité de recherche INRIA Sophia Antipolis : 2004, route des Lucioles - BP 93 - 06902 Sophia Antipolis Cedex (France) 